\title{
The Russian Version of the Oxford Cognitive Screen: Validation Study on Stroke Survivors
}

AQ: au

AQ: 1
Maria Shendyapina

University of Hong Kong

Sergey Kazymaev

Treatment and Rehabilitation Center of the Ministry of Healthcare of Russia, Moscow

\author{
Ekaterina Kuzmina \\ University of Oslo
}

Anna Petrova

University of Hong Kong

Brendan S. Weekes

University of Hong Kong

\author{
Nele Demeyere \\ University of Oxford
}

\begin{abstract}
Objective: The Oxford Cognitive Screen (OCS) is a screening tool for the assessment of poststroke deficits in attention, memory, praxis, language, and number processing. The goal of the present study was to develop a Russian version of the OCS (Rus-OCS) via translation of the original battery, its cultural and linguistic adaptations, and reporting preliminary findings on its psychometric properties. Method: All parts of OCS were translated by native Russian-speaking neuropsychologists. Russian-speaking stroke patients $(N=205)$ were assessed with the Rus-OCS. Their performance was compared with performance of 60 healthy Russian-speaking adults aged between the ages of 18 and 91 years. The performance of 15 stroke patients and 42 healthy adults were assessed with a parallel version within 7 days of first testing. Convergent validity of the Rus-OCS was established via correlations with comparable tasks. Performance of three stroke groups with different lesion lateralization (right, left, and bilateral) was compared on language and visual attention subtasks. Preliminary normative data based on 5th to 95th percentile were also reported. Results: Measures of internal consistency and test-retest reliability ranged from acceptable to very good and estimates of convergent validity ranged from moderate to high. Sensitivity and specificity was found to range from .56 to 1 and from .73 to 1, respectively. Significant differences in performance between stroke and healthy groups on all subtasks confirmed the discriminative power of the Rus-OCS was good. Conclusions: Rus-OCS is a promising cognitive screening instrument for Russian-speaking patients. However, further validation is needed. Constraints of socioeconomic differences between Russian speakers in the wider population should be considered.
\end{abstract}

Maria Shendyapina, Laboratory for Communication Science, Faculty of Education, University of Hong Kong; Ekaterina Kuzmina, Center for Multilingualism in Society across the Lifespan, Faculty of Humanities, University of Oslo; Sergey Kazymaev, Department of Neurorehabilitation, Treatment and Rehabilitation Center of the Ministry of Healthcare of Russia, Moscow, Russian Federation; Anna Petrova, Laboratory for Communication Science, Faculty of Education, University of Hong Kong; Nele Demeyere, Department of Experimental Psychology, Cognitive Neuropsychology Centre, University of Oxford; Brendan S. Weekes, Laboratory for Communication Science, Faculty of Education, University of Hong Kong.

Maria Shendyapina contributed to this project by obtaining ethical approval; drafting the manuscript; and performing data analysis and interpretation, data collection, and statistical analysis. Ekaterina Kuzmina contributed by revising the manuscript, designing the study concept, and translating the subtests. Sergey Kazymaev contributed by obtaining the access to the clinical database and performing data collection and data analysis. Anna Petrova contributed by translating the subtests and designing the study concept. Nele Demeyere contributed by revising the manu- script. Brendan S. Weekes contributed by revising the manuscript and supervising the study.

This article is based on data used in the doctoral dissertation of Maria Shendyapina, which was supported by the Research Grants Council Hong Kong PhD Fellowship Scheme.

We acknowledge the contribution that Professor Glyn Humphreys made by inspiring many local clinical and scientific communities in different countries to pursue studies of cognitive assessment in neurological diseases. Professor Humphreys passed away on January 14, 2016 after fruitful discussions and consultations on the current project.

To use the OCS, researchers should contact the copyright owners, Oxford University Innovation, either by e-mail at healthoutcomes@ innovation.ox.ac.uk or by applying via the online license request portal at https://process.innovation.ox.ac.uk/clinical

Correspondence concerning this article should be addressed to Maria Shendyapina, Laboratory for Communication Science and Faculty of Education, University of Hong Kong, Pokfulam, Hong Kong SAR. E-mail: mshend@hku.hk 


\section{General Scientific Summary}

The present study describes the adaptation and validation of a stroke-specific cognitive screening tool called the Rus-OCS in a Russian-speaking sample. Introducing this tool responds to the need of quantitative neuropsychological screening tools available in Russian. Our study provides a picture of poststroke cognitive deficits which are not language-specific drawn from relatively large clinical sample $(N=205)$.

Keywords: cognitive testing, neuropsychological assessment, stroke, Russian, validation

Stroke leads to a variety of cognitive deficits affecting the quality of life (Carod-Artal, Egido, González, \& De Seijas, 2000), personal relationships (Thompson \& Ryan, 2009), mood (Carson

AQ: 3 et al., 2000), activities of daily living and overall physical function (Corbetta et al., 2015; Lai, Studenski, Duncan, \& Perera, 2002). In the case of minor stroke or transient ischemic attack causing relatively small lesions, there may be no obvious visible symptoms

AQ: 4 (Easton et al., 2009). However, severe lesions tend to result in a broader range of sensory, motor, and cognitive impairments (Barker-Collo \& Feigin, 2006), including memory, executive control, and language deficits as well as visuospatial neglect (Moorhouse \& Rockwood, 2008; Patel, Coshall, Rudd, \& Wolfe, 2002). Neuropsychological diagnostic tools can discriminate domaingeneral deficits (e.g., decreased executive control) from domainspecific ones (e.g., aphasia or visuospatial neglect), necessary for planning patients' cognitive rehabilitation (Cumming, Marshall, \& Lazar, 2013; Massa et al., 2015). Furthermore, individual profiles of cognitive impairments provide information for treatment planning (Cumming et al., 2013) and predict recovery outcomes (Nys et al., 2005). Therefore, importance of having reliable and valid instruments for poststroke cognitive screening cannot be overestimated.

\section{Cognitive Screening in Stroke}

Cognitive screening instruments require, at a minimum, acceptable reliability and validity. Most cognitive screening tests developed to detect dementia, such as the Montreal Cognitive Assessment (MoCA; Nasreddine et al., 2005), Mini-Mental State Examination (MMSE; Bour, Rasquin, Boreas, Limburg, \& Verhey, 2010), and Addenbrooke's Cognitive Examination Revised (ACE-R; Morris, Hacker, \& Lincoln, 2012), all have at least adequate validity and reliability. However, tests of cognitive decline are not necessarily valid for cognitive assessment following stroke (Dong et al., 2010; Pendlebury, Mariz, Bull, Mehta, \& Rothwell, 2012). This is because dementia screening instruments do not consider in detail the limiting effects of poststroke functional impairments to attention and language on test performance raising the likelihood that any residual cognitive strengths may be masked. This reduces the validity of a screening test for profiling potential cognitive functions following stroke (Demeyere, Riddoch, Slavkova, Bickerton, \& Humphreys, 2015). For example, aphasia after stroke is often a constraint on performance when given neuropsychological tests despite preserved cognitive processing. Some screening tools include nonverbal subtasks to reduce such constraints on the performance of patients with language difficulties. However, extant instruments do not typically allow patients to provide nonverbal responses on all tasks. For example, on MoCA orientation task patients with expressive aphasia will score zero even when they can perform the task correctly with a nonverbal response such as pointing to the correct date on a calendar via forced choice. Similarly, a patient with visuospatial neglect may not attend to graphic stimuli presented in the neglected side of space despite preserved recognition of stimuli with attentional cues.

Current screening tools for poststroke cognitive function are also limited by often exclusive assessment of one cognitive domain (attention, language, memory) without concurrent testing of domain general impairment using an equivalent and standardized format. Therefore, even if a test is highly reliable and valid, the results assess performance within a relatively narrow range of cognitive function, for example, language (Kuzmina, Humphreys, Riddoch, Skvortsov, \& Weekes, 2018). Assessment of intact and impaired cognitive function using a standardized test battery increases the sensitivity of a neuropsychological screening instrument. Ideally, tools will also be independent of language specific constraints to increase validity for use across cultures and socioeconomic classes (e.g., Raven, 2000). The advantage of language independent screening tools is replicability across countries and cultures, thus providing an instrument with universal application for testing neuropsychological processing (see Kong et al., 2016, 2017; Kuzmina et al., 2018). Such a tool has advantages beyond simply neuropsychological diagnosis and rehabilitation in a world where cultural and linguistic diversity is increasing (Abutalebi \& Weekes, 2014). Big data sets collected across cultures, ethnicities and languages are now shared for development of theories and treatments in a range of neurological disorders and in neuroimaging methods, for example, in the Evidence-based Network for the Interpretation of Germline Mutant Alleles project (i.e., ENIGMA; Thompson et al., 2014).

Reviews of cognitive screening instruments (Cullen, O'Neill, Evans, Coen, \& Lawlor, 2007; Hachinski et al., 2006; Dong et al., AQ: 5 2010) have identified several different types of diagnostic measures. Brief screening scales, including MoCA (Nasreddine et al., 2005), Neurobehavioral Cognitive Status Examination (Mysiw, Beegan, \& Gatens, 1989), MMSE (Bour et al., 2010), ACE-R (Morris et al., 2012), Repeatable Battery for the Assessment of Neuropsychological Status (Wagle et al., 2011), the Cognitive Functional Independence Measure (Zwecker et al., 2002), and the Cambridge Cognition Examination (CAMCOG; de Koning et al., 1998). These scales are time-efficient, have short administration 
(between 10 and $30 \mathrm{~min}$ ) and can be used for inpatient admission and outpatient evaluations. However, a criticism of such scales is they do not classify the properties of a tested domain in detail using established terms derived from cognitive neuropsychology, for example, the type of memory impaired (episodic vs. semantic), the locus of language impairment (word or sentence), and auditory versus visual comprehension, nor do they test the limits of a cognitive process that is, provide opportunities to demonstrate correct performance for example, line drawing with apraxia.

In response to these limitations, stroke- and aphasia-specific neuropsychological batteries have been developed. These include the Birmingham Cognitive Screen (Humphreys, Bickerton, Samson, \& Riddoch, 2012), the OCS (Demeyere et al., 2015, 2016), the Cognitive Assessment Scale for Stroke Patients (CASP; Barnay et al., 2014), Western Aphasia BatteryRevised (Kertesz, 2006), Cognitive Linguistic Quick Test-Plus (CLQT+; Helm-Estabrooks, 2001), and the National Institute of Neurological Disorders and Stroke-Canadian Stroke NetAQ: 6 work Vascular Cognitive Impairment (NINDS-CSN VCI) Neuropsychological Assessment Protocol (Han, Anderson, Jones, Hermann, \& Sattin, 2014).

Although these instruments are more suitable for testing the limits of neuropsychological processes in stroke, one limitation of these instruments is that most of them have been validated on limited samples. Inclusivity, sensitivity and specificity of such instruments are therefore dependent on the size and type of clinical group. Ideally, a valid instrument will be sensitive to a wide range of neurological conditions. However, these instruments can help to identify the most important patterns of functional and cognitive abilities in any patient to form a unique profile for subsequent more detailed assessments (Cullen et al., 2007).

Questionnaires can be applied to a larger sample and a wider range of neuropsychological conditions. For example, the Stroke Impact Scale (Richardson, Campbell, Allen, Meyer, \& Teasell, 2016), the Telephone Interview for Cognitive Status (Barber \& Stott, 2004), and the Informant Questionnaire on Cognitive Decline in the Elderly (McGovern, Pendlebury, Mishra, Fan, \& Quinn, 2016) are all reliable and valid self-report instruments. The aforementioned questionnaires also have validity, so they can be used in community care programs, and they can often be executed via telecommunication services online, by telephone, or by caregiver reports. However, subjective reports of informants are prone to human error, and interpretation could be limited by biases.

Computerized assessments reduce human error and subjectivity. These instruments include OCS + (Humphreys et al., 2017), CogState Brief Battery (Maruff et al., 2009), Cambridge Neuropsychological Test Automated Battery (Egerházi, Berecz, Bartók, \& Degrell, 2007), Cognistat Assessment System (Mueller, Kiernan, \& Langston, 2011) and others. Most of them are automatized adaptations of self-report and paper-and-pencil diagnostic tools, although the software also allows a deeper level of data analyses for example, via deep learning to predict outcomes using objective measures such as motor speed, reaction time, eye tracking and EEG. In addition, online computation of testing improves the validity, reliability, sensitivity and standardization of instruments across different age groups, levels of education, and socioeconomic status (Zygouris, \& Tsolaki, 2015). Potential bias can occur in computerized assessment because of varying levels of computer literacy and use of technology (particularly in seniors), as well as learning effects that emerge in repeated measures (Parsons, 2016). It has also been argued that computerized assessments do not solve the core problem of ecological validity that characterize neuropsychological testing (though see Burgess et al., 2006). These problems aside, adaptation of big data, neural interfaces and virtual reality to neuropsychological screening is likely the next generation of neuropsychological assessment.

Comprehensive neuropsychological assessment use domain specific tests measuring highly refined cognitive functions. The Frontal Assessment Battery (Oguro et al., 2006), Neuropsychological Assessment Battery (Stern \& White, 2003), and Delis-Kaplan Executive Function System (Delis, Kramer, Kaplan, \& Holdnack, 2004) are typical of cognitive neuropsychological case reports. Such assessments are widely recognized as important for planning cognitive rehabilitation (see Robinson \& Weekes, 2013), because they adopt a bespoke diagnostic system consisting of statistically robust and clinically valid instruments that are tailored to the individual case. On the other hand, use of too many alternative tests makes comparison between different clinical groups difficult, and relies ultimately on choices of a neuropsychologist that can cause subjective biases.

\section{The OCS}

The OCS (Demeyere et al., 2015) was designed to assess poststroke cognitive deficits. The key features of the OCS are to minimize demands on language production and visuospatial processing in order to increase test sensitivity to cognitive deficits in five main cognitive domains identified in the neuropsychological literature: executive control, calculation, number writing, memory, language, and praxis. A distinctive feature of the OCS is all subtasks can be completed with one hand thus reducing the influence of upper limb hemiparesis after stroke. Also, OCS was specifically designed to be inclusive of patients with aphasia and visual neglect. It is constructed from high frequency short words presented orally and visually on a printed booklet, so the patient can reply by pointing instead of producing oral responses. Furthermore, the tasks have been designed to reveal independent cognitive processes in different trials. For example, items in picture naming, picture pointing, and sentence reading subtasks are repeated to test language as well the integrity of memory.

Administration of the OCS is relatively fast, that is, approximately $15 \mathrm{~min}$, and testing results are summarized in a visual snapshot, or wheel of cognition. The OCS developed for the United Kingdom (UK-OCS) has been adapted and validated in other languages, such as Cantonese (Kong et al., 2016, 2017; Lam, Kong, Ho, Humphreys, \& Weekes, 2014), Italian (Mancuso et al., 2016, 2018), and Putonghua (Hong et al., 2018), suggesting utility for greater poststroke cognitive screening globally. The development of alternative language versions of the OCS for example, in Hong Kong (HK-OCS) followed the original OCS validation process closely and resulted in normative scores across three age groups and education levels for Cantonese speakers with very good to excellent values for validity, reliability, and internal consistency. In terms of discriminative validity, Kong and colleagues (2016) reported an excellent value of the HK-OCS to differentiate stroke patients from neurologically intact, healthy adults. Mancuso and colleagues (2016) reported norms for healthy Italian speakers adjusted for age, education, and gender. Hong et al. (2018) re- 
cently published validation study of the Oxford Cognitive Screen with Putonghua speakers in Mainland China (OCS-P) using more advanced statistical methods than previous adaptations. There were psychometric properties reported for younger and older healthy groups and subacute stroke survivors similar to other studies including satisfactory levels of validity, reliability and discriminative properties of the OCS-P (see the Appendix in the online supplementary material for full comparison of OCS validation procedures with different languages).

\section{Cognitive Screening Assessment in Russia}

Development of cognitive screening tools in clinical settings was one of the most important goals of the Soviet school of neuropsychology (Luria, 1966; Luria \& Hutton, 1977; Luria \& Majovski, 1977). However, the majority of assessment tools developed in Russian have remained predominantly qualitative and lack rigorous testing of their reliability, sensitivity, and validity (although see Ivanova \& Hallowell, 2009; Kuzmina et al., 2018). Akhutina and Melikyan (2012) argued that a scarcity of quantitative neuropsychological assessment tools for Russian speakers was long standing. Luria assumed that

\footnotetext{
. . . higher mental functions may exist only as a result of interaction between highly differentiated brain structures and that each of these structures makes its own specific contribution to the dynamic whole and plays its own role in the functional system. (Akhutina, 2015, p. 879)
}

According to Luria's syndrome-based approach, each part of a neuropsychological examination should be a separate experiment where a scientist tests theory driven hypotheses about causes of observed cognitive deficits (Luria, 1966). On the basis of this approach, the Lurian Neuropsychological Battery (LNB; Luria, 1966) was developed for testing with a consequent adaptation to the Luria-Nebraska Neuropsychological Battery (LNNB; Golden, 1980). Despite the adaptation and continued widespread use of the LNB in Russia and LNNB in the United States, there is a lack of time-efficient screening instruments that are appropriate for clinical practice in Russia (Akhutina \& Melikyan, 2012). One consequence of the slow development of standardized neuropsychological tools in Russia is that studies of Russian speakers with neuropsychological impairments have limited impact since there are no valid norms of the established cognitive evaluation procedures. It has been reported that Russian clinicians use cognitive screens that were developed in other linguistic and cultural settings without adaptation, validation and standardization for Russian speakers (Rasskazova, Kovyazina, \& Varako, 2016). For example, Russian versions of the MoCA (Makeeva et al., 2012) and MMSE (Levin et al., 2015) are available. However, there are no normative or standardized guidelines published for use with the Russian clinical population. This is suboptimal considering the demands of parameters for evidence based assessment, intervention and research in neuropsychology are growing across the world. Thus, the development of a linguistically and culturally adapted cognitive screen with established psychometric properties for the Russian language would be an advantage for clinicians aiming to deliver standardized neuropsychological diagnostics.

\section{Aims and Hypothesis}

Our aim was to develop a reliable, sensitive, and valid poststroke assessment tool for Russian-speaking patients by adapting the OCS into Russian (Rus-OCS). To do this, we (1) modified OCS stimuli into culturally and linguistically valid items for Russian speakers; (2) collected reliability, convergent validity, sensitivity, specificity data, and preliminary normative score values based on 5th and 95th percentiles for this population; and (3) compared performance of neurologically impaired participants with different lesion locations (left vs. right) to estimate discriminative power. Selected data are reported here to illustrate the potential of the Rus-OCS for use with typical Russian speakers. Our hypothesis was that Rus-OCS would generate responses that discriminate between Russian speakers with and without cognitive impairment following stroke and more critically provide individual profiles of cognitive impairment with widely acceptable psychometric properties. On the basis of previous studies (Demeyere et al., 2015; Kong et al., 2016), we also predict that patients who have left hemisphere lesions ( $\mathrm{LH})$ will perform worse on subtasks that require language and verbal functions compared with patients who have right hemisphere lesions $(\mathrm{RH})$ who will conversely perform worse than $\mathrm{LH}$ patients on subtasks testing visuospatial functions.

\section{Method}

\section{Participants}

The background demographic and clinical details of 205 participants who had a stroke and 60 healthy participants are summarized in Table 1. The stroke participants were recruited from the T1 neurological department of the Treatment and Rehabilitation Center of the Ministry of Healthcare of the Russian Federation according to the following inclusion criteria: (a) premorbid right-handedness; (b) absence of comorbidity with any other health condition of neurological or mental illnesses and severe somatic diseases (e.g., diabetes or intense chronicle pains); (c) absence of severe difficulties in comprehending instructions and/or inability to maintain attention for at least $30 \mathrm{~min}$; (d) absence of significant hearing and/or visual deficits; and (e) presence of cognitive deficits (based on the neurological admission tests). All patients were assessed by neurologists, optometrists, neuropsychologists, and other health specialists upon admission to the Center. The ability of patients to comprehend instructions was based on comprehensive neurological assessment developed in the Center and administered by specialists and therefore included into all patients' clinical notes.

The sample ranged in age from 18 to 88 years old and comprised 85 women $(M=63.96, S D=13.87)$ and 120 men $(M=60.32$, $S D=16.91)$. Their poststroke time ranged from 0 to 123 months $(M=8.44, S D=19.12)$; there were 32 patients in the acute state ( 0 to 6 days post onset), 116 patients in the subchronic state (1 to 5 months post onset), and 56 patients in the chronic state (6 to 123 months post onset).

On the basis of information from MRI scans reported in patients' clinical notes, the whole clinical sample was divided into three subgroups with a different location of lesion: left-hemisphere (LH; $n=41$ ), right-hemisphere (RH; $n=46$ ), and bilateral (BL; $n=118)$ stroke patients. There were no significant differences between subgroups in mean age, years of education and gender 
balance. The only significant difference was in months postonset observed between the LH-RH subgroups which was caused by one to three outliers who were more than 120 months poststroke in the $\mathrm{RH}$ and BL groups (the latter not significant).

As expected, some patients in the clinical subgroups were diagnosed with aphasia. There were $61 \%$ of LH patients $(n=25$; four with sensory aphasia, eight with motor aphasia, 12 with mixed aphasia, and one with amnestic aphasia), $13 \%$ of RH patients ( $n=$ 6 ; all with motor aphasia), and $9 \%$ of BL patients $(n=11$; three with motor aphasia, five with mixed aphasia, and three with amnestic aphasia) had language impairments reported in their medical records. Further testing confirmed these observations.

Healthy participants were aged between 20 and 91 years including 40 women $(M=61.60, S D=18.99)$ and 20 men $(M=59.85$, $S D=18.50)$ all recruited from the Moscow region. Additional analyses of the distribution of education levels across different ages in the healthy group found no significant differences according to sociodemographic data extracted from databases from the official website of the Russian Federal State Statistics Service (see http://www.gks.ru/bgd/regl/b12_13/IssWWW.exe/Stg/d2/07-03 $. \mathrm{htm})$; that is, the healthy group represented the typical distribution of age-ranges of all Russian citizens.

Exclusion criteria were identical to the patient sample. The visual and hearing acuity of the healthy sample was verified by oral report from participants. There were no significant differences between groups in handedness (all right-handed), mean age or level of education. One difference between groups was in their gender composition (see Table 1), and this is acknowledged as a limitation of the present study.

All of the participants were informed of the protocol, procedure and outcomes of the research before they signed an informed consent form giving agreement to participate. Participation did not involve any material compensation. However, a detailed feedback report on the testing results was provided upon request. All experimental procedures including informed consent for participation in the protocol were approved by the Human Ethics Committee of the University of Hong Kong (HREC Reference Number: EA17-07009). As the participants were recruited from the Moscow Rehabilitation Center, a specific consent for conducting the research and collecting medical records was also obtained through the local ethics committee of the Center.

\section{Materials}

All participants were given the Russian version of MoCA (Makeeva et al., 2012), the LNB (Luria, 1966), and Star Cancellation Test (SCT; Friedman, 1992) together with the Rus-OCS Version A. Normative data were taken from official websites for MoCA and SCT although we note that neither website provided language or cultural specific norms for the Russian population and all used norms were derived from English speakers with translations. The SCT was adapted for the Russian population by replacing English letters and words with the same length Russian stimuli (see Figure 1), according to recommendations from the official SCT website which states the following: "the words can be translated into the patients' native language" (see https://www.strokengine.ca/quick/ sct_quick/).

Choice of the validation tasks was inspired by previous studies (Demeyere et al., 2015; Kong et al., 2016). MoCA was used in 


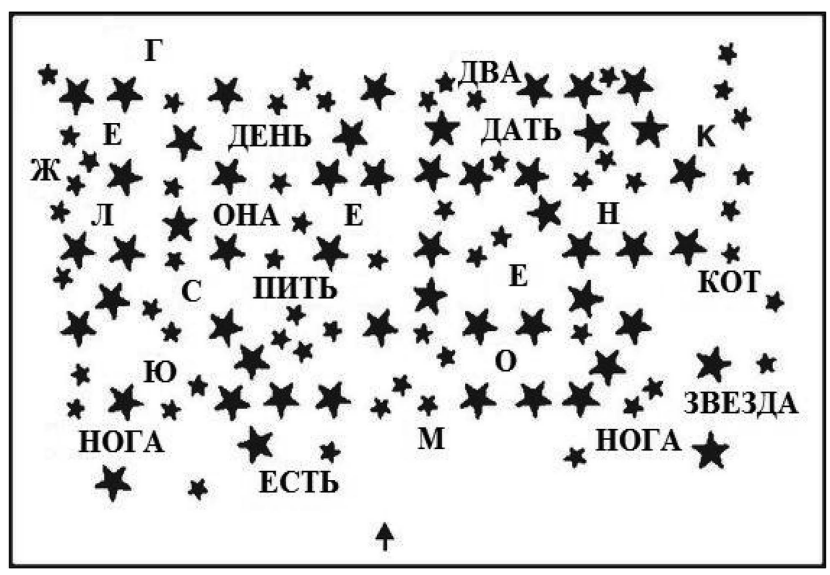

Figure 1. Russian version of the Star Cancellation Test.

both abovementioned studies; the gestural production test was used in the HK-OCS validation and is similar to both the LNB Dynamic Kinesthetic Praxis and the SCT used in the UK-OCS. The length of a testing session with Rus-OCS and all validation tasks (including the instructions, explanations, and other formal procedures) was approximately $1 \mathrm{hr}$ on average, allowing a break between sessions. The testing of the clinical sample was complemented with neuropsychological case reports derived from Lurian assessment protocols taken from the medical database of the Center together with patients' MRI scans confirming the stroke location. Relevant data were extracted and used in the psychometric analyses.

Forty-two healthy participants and 15 stroke patients were retested with Version B within 7 days of testing with Version A $(M=4.2, S D=1.49)$. Both Versions A and B comprised subtasks measuring identical cognitive domains, with alternative content. For example, four pictures from the Picture Naming task representing hippopotamus, watermelon, flamingo, and pear in Version A were replaced by pictures of a spanner, bear, zebra, and carrot in Version B.

The retest verification for the majority of the stroke sample was not possible because of the intensive cognitive rehabilitation program established in the Center which causes a serious bias in the retest values even within 3 days. The 15 patients added into analyses of test-retest reliability were on the waiting list for the beginning of their rehabilitation program which allowed them to be tested before their cognitive training had commenced.

\section{Translation and Cultural-Linguistic Modifications of the OCS to Russian}

A majority of cognitive assessment tools is initially developed and validated in Western culture and for English speakers only while being adapted directly and without the cultural considerations necessary for use in other languages. Although, it is impossible to apply norms from any test that is developed in a different culture and language because of obvious linguistic and cultural diversity. A more valid approach is to develop screening instruments that do not depend on knowledge of culture and do not rely on verbal responses in one language only (Kuzmina et al., 2018).
Thus, the cognitive study of neuropsychological impairments in the patients who speak the Russian language requires careful translation and cultural adaptation. There are many linguistic features unique for Russian language in comparison with English (Panchenko et al., 2018). For example, Russian is written in the Cyrillic alphabet consisting of 33 letters. Russian grammar has a synthetic morphology and syntax with three genders and six cases. The sentence structure differs from English by use of flexible word order representing semantic meaning (Wierzbicka, 1997). Previous studies on the adaptation of the Birmingham Cognitive Screen to the Russian language (Kuzmina et al., 2018) revealed the importance of following the logic of the initial testing protocol, choosing high-frequency words (except in cases where word frequency is one of the testing parameters) and use of backward translation that is, Russian text translated into English by native Russian-English speakers and vice versa as a check of a general translation adequacy.

Two parallel versions of the OCS (A and B) were translated into Russian. Given that English and Russian are both Indo-European languages and the sociocultural background of Russian speakers in Moscow is now very similar to Western culture in many aspects, it was expected that straightforward translation would be suitable for most of the instructions and tasks which can be considered language-neutral (e.g., drawing lines in the Executive task, crossing hearts in the Broken Hearts subtask, or performing calculations on the Calculation subtask). In total, translation of stimuli was necessary in six out of 12 subtasks and one subtask of Picture Naming requiring the replacement of a single word item. The logic of translation and cultural-linguistic adaptation are explained subsequently.

All subtasks required the translation of instructions. Original AQ: 8 instructions from the OCS (English) were composed of simple sentences with high-frequency short words. The same principles were applied to the instructions used in the Rus-OCS which were translated straightforwardly. All instructions were then backtranslated from Russian into English by three native RussianEnglish speakers ensuring the resulting back translation was equivalent in meaning to the original OCS text as closely as possible (no errors were noted). The same procedures were applied to the translation of the Rus-OCS parallel Version B.

The Picture Naming subtask originally consisted of four pictures: hippo/hippopotamus, melon/watermelon, filing cabinet/ chest of drawers, pear. Because of the absence of a Russian word for filing cabinet, the members of the UK-OCS team suggested replacement with the item flamingo, which was consistently retrieved by a majority of Russian speakers. Replacement of other items in Version A and all items in Version B (spanner, bear, zebra, carrot) was not necessary because words had translations in Russian.

The Picture Pointing subtask included four picture items: tool, fruit, vegetable, animal in Version A and farm animal, wild animal, fruit, tool in Version B. All pictures categories were familiar for Russian population, so no item replacement was needed.

In the Orientation subtask, there were four questions about time and place where the participant is required to say or point on the booklet in which city he or she is right now, what part of the day it is now, as well as in which month and year we are now. In the multiple choice booklet, the English city names were replaced with Russian city names that are similar in terms of size and sociopo- 
litical status. Authors of the OCS (Demeyere et al., 2015) recommended using the following logic of cities replacement: $1=$ correct answer; 2 = known city of the same size; 3 = nearest city of the same size; and $4=$ known city located nearby.

Although the time of the day and the names of months were translated straightforwardly, the items for choice of year were replaced again by the logic provided by Humphreys and colleagues (2012), whereby $1=199 x$ ( $x$ is the last digit of the current year); $2=$ current year $+1 ; 3=$ current year -1 ; and $4=$ correct answer.

In the Sentence Reading subtask, sentences including four words (in italics) that cannot be read correctly without lexical knowledge were used to detect the signs of surface dyslexia: "Интересно, какой счет будет в конце игры, - подумал радостный мужчина, щурясь от яркого утреннего солнца" [Interestingly, what score will be in the end of the game, the cheerful man wondered, squinting because of the bright morning sun]. Although Russian is known to be a relatively transparent language for oral reading, that is, the mappings between orthography and phonology are regular, some words cannot be read correctly via regular grapheme/phoneme mappings (Kornev, Rakhlin, \& Grigorenko, 2010; Ulicheva, Coltheart, Saunders, \& Perry, 2016). For instance, the letter $\mathrm{m}$ [t] in the word радостный (cheerful [radosnyj]) is omitted during oral reading. In the word cyem (score [schyot]), the letters $c$ and $y$ should be read as one sound [shch] instead of separate sounds corresponding to the letters $c[\mathrm{~s}]$ and $\psi[\mathrm{ch}]$. The Reading subtask included such words to screen for surface dyslexia. The use of irregular words allowed for screening of reading ability and potentially premorbid level of educational and intellectual function poststroke. Table 2 summarizes the stimuli that were included in the Sentence Reading subtasks of the parallel versions to reveal disturbances to the lexical route and signs of surface dyslexia in Russian.

The subtasks of Visual Field, Number Writing, Calculation, Broken Hearts, Gesture Imitation, and Executive functions required direct translation of the instructions only because of the nonverbal nature of the testing stimuli. There were no changes in the stimuli in Episodic Recognition because all pictures were well-known for Russian population and the correct answers were matching with the unchanged items of the picture pointing subtask.

The Verbal Recall and Recognition subtask stimuli were replaced on advice of OCS creators: On each trial there was one correct word, one synonym to the correct word, one word which sounds similar to the correct word, and one word with similar meaning with the correct word.

\section{Data Analysis}

The workflow of the present study was very similar to extant validation studies of the OCS (Demeyere et al., 2015; Kong et al., 2016; Hong et al., 2018; see also the Appendix in the online supplementary material). The Kruskal-Wallis test was used to contrast performance of stroke patients with healthy participants on the Rus-OCS subtasks. In addition, we calculated the values of the 5th and 95th percentiles of the performance of healthy participants to estimate preliminary cut-off scores and compare these with reported cut-offs from previous studies (Demeyere et al., 2015). For most of the subtasks, impairment means that the patient's score is lower than the normative value based on the 5th percentiles from the healthy sample. However, in the Asymmetry subtask measuring visuospatial neglect, scores higher than the normative score are also evidence of neglect. The same is true for the Executive subtask score which represents the subtraction of scores on the shifting trail task from the sum of two simple trail tasks. Hence, a score that is larger than the normative cutoff on this subtask signifies weaker performance for the shifting condition that is, the test of cognitive control. To assess possible effects of demographic variables on scores, normative values for three age groups ( $<50$ years old, 50 to 69 years old, $>69$ years old) and two levels of education (secondary and tertiary) were calculated for each subtask. To check the internal consistency of translated subtasks, Cronbach's alpha coefficients (Cronbach, 1951) were calculated. Test-retest reliability was established for the healthy sample and for a separate group of 15 stroke survivors (prerehabilitation as discussed earlier), because other patients were enrolled in cognitive rehabilitation programs (each patient received at least three training sessions per day), which could influence the test-retest results. Convergent validity was investigated via correlations between patient scores for Rus-OCS subtasks and scores for Rus-MoCA subtasks as well as relevant subtasks from the SCT and LNB. To check the sensitivity and specificity of Rus-OCS, MoCA, SCT, and LNB were used. Since there are no Russianspecific norms reported for MoCA or SCT, all diagnostic outcomes were additionally supported by results of comprehensive neuropsychological assessments taken from each patient's medical records. In addition, we compared the incidence of impairments in the clinical sample overall $(N=205)$ and impairments according to different lesion locations. We then compared patients with first stroke in the left hemisphere $(n=41)$ or right hemisphere $(n=46)$ to a group with bilateral lesions $(n=118)$. The Kruskal-Wallis test was used to test for differences between clinical groups in perfor-

Table 2

Irregular Words Used in the Rus-OCS Sentence Reading Subtask

\begin{tabular}{|c|c|c|}
\hline Word & Spelling [Pronunciation] & Irregularity \\
\hline 1А. счёт, score & schot $[$ shch ̌ot $]$ & Two letters $s$ and $c h$ are pronounced as one sound $[s c h]$ instead of $[s]$ and $[c h]$ \\
\hline 2А. радостный, cheerful & radostnyi [radosnȳi] & $T$ is not pronounced \\
\hline 3А. мужчина, man & muzhchina [mushchina] & Two letters $z h$ and $c h$ are pronounced as one sound $[s h c h]$ instead of $[z h]$ and $[c h]$ \\
\hline 4А. солнца, sun & solntsa [sontsa] & $L$ is not pronounced \\
\hline 1В. что, what & chto $[$ shto $]$ & $S h$ is pronounced instead of $c h$ \\
\hline 2В. нового, new & novogo $[$ novova $]$ & Go is pronounced as $v a$ \\
\hline 3В. мужчина, man & muzhchina [mushchina] & Two letters $z h$ and $c h$ are pronounced as one sound $[s h c h]$ instead of $[z h]$ and $[c h]$ \\
\hline 4В. мягкое, soft & myagkoye [myakhkoře] & The voiced consonant sound $g$ is substituted by its voiceless pair sound $[k h]$ \\
\hline
\end{tabular}

Note. $\quad$ Rus-OCS $=$ Russian version of the Oxford Cognitive Screen. 
mance on all Rus-OCS subtasks. Additionally, the chi-square test was used to check for equality in the proportion of impairments on subtasks between clinical groups. Although the OCS is not directly designed for topical neurological diagnostics, the clinical profiles presented here may be helpful for the interpretation of individual results in practice.

\section{Results}

\section{Group Differences and Preliminary Cut-Offs}

Performance on all Rus-OCS subtasks was significantly different between healthy and stroke groups (see Table 3). Comparison of the preliminary Rus-OCS and UK-OCS cut-offs revealed minor differences in the Sentence Reading task (which was completed with $100 \%$ accuracy by all healthy participants) whereas a single mistake was considered normal range performance for the UKOCS. At the same time, in the Broken Hearts subtask, the range of normative accuracy $(\geq 40)$ was slightly lower than in the U.K. sample $(\geq 42)$ and the range of scores on the Space Asymmetry subtask was more balanced (from -3 to 3 ) than in the UK-OCS (from -2 to 3 ). Another divergent value was detected on the accuracy cutoff score for the Executive Function subtask, which was two points higher in the Russian sample (i.e., 6) than the U.K. sample (i.e., 4). All differences will be further discussed in the Discussion section.

Descriptive statistics for each age cohort are summarized in Table 4. Age-related differences for the healthy group were observed for all subtasks except for Picture Pointing and Sentence Reading subtasks, which both produced a ceiling effect. In the stroke group, there were no age-related differences on any memory subtask (Verbal Recall and Recognition, Episodic Recognition) or the Executive Function subtask scores. In the healthy group, agebalanced groups with lower educational levels had reduced scores on all subtasks except for Picture Pointing, Sentence Reading, Number Writing, and Verbal Recall. There were no significant differences between females and males on any subtask for either group $(p<.05)$. In the healthy group, there were significant correlations $(p<.001)$ between age and performance on all subtasks that did not produce ceiling effects: Picture Naming $\left(r_{\mathrm{p}}=-.44\right)$, Orientation $\left(r_{\mathrm{p}}=-.37\right)$, Broken Hearts $\left(r_{\mathrm{p}}=-.42\right)$, Gesture Imitation $\left(r_{\mathrm{p}}=-.64\right)$, Verbal Recall $\left(r_{\mathrm{p}}=-.81\right)$, Verbal Recognition $\left(r_{\mathrm{p}}=-.64\right)$, and Episodic Recognition $\left(r_{\mathrm{p}}=-.60\right)$, Executive Score $\left(r_{\mathrm{p}}=.51\right)$, and the Mixed Executive task $\left(r_{\mathrm{p}}=-.57\right)$. Correlations for the Rus-OCS subtask scores with gender and years of education were not significant (all $p \mathrm{~s}>.05$ ).

\section{Internal Consistency}

Cronbach's alpha was calculated for language scales that contained replacement items only: Sentence Reading $(\alpha=.89)$, Picture Naming $(\alpha=.77)$, Verbal Recall $(\alpha=.82)$, and Verbal Recognition $(\alpha=.83)$. Other scales from the OCS battery (e.g., pictures of hearts in the Broken Hearts subtask, pictures of circles and triangles in the Executive functions subtask, numbers in the Calculation subtask) were not translated and thus satisfactory consistency was assumed.

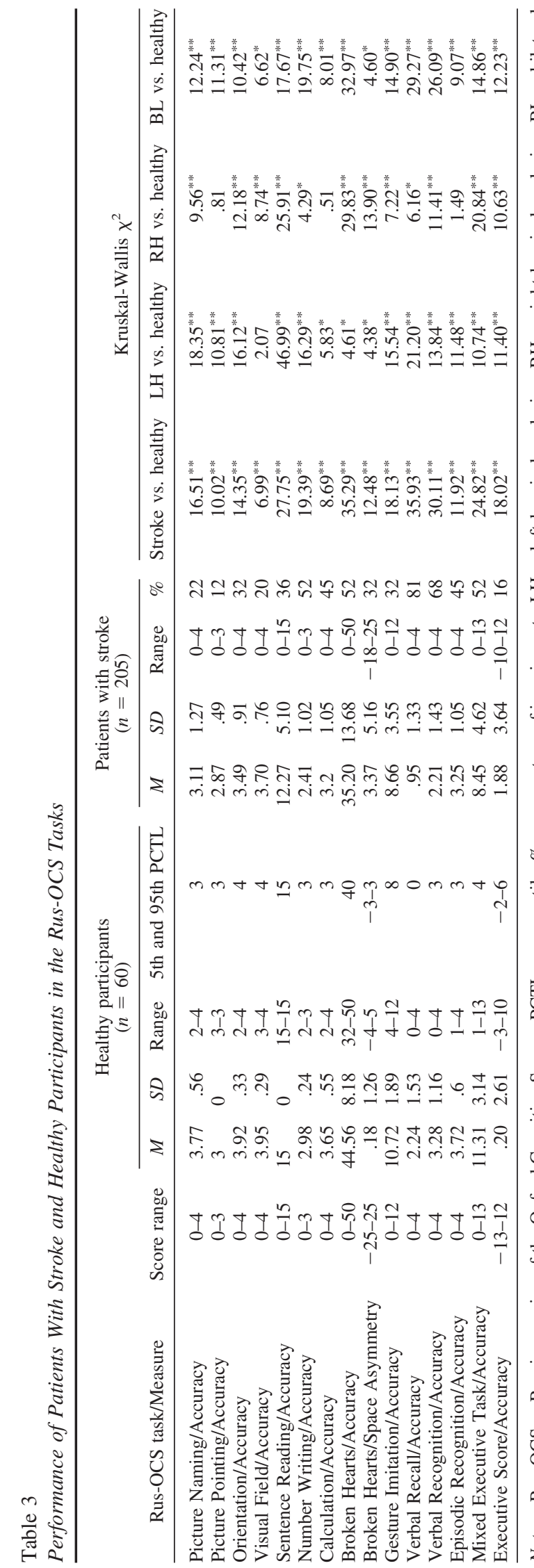


Table 4

Variation of the Rus-OCS Scores by Age and Education

\begin{tabular}{|c|c|c|c|c|c|c|c|c|c|c|}
\hline \multirow[b]{3}{*}{ Rus-OCS task/Measure } & \multicolumn{5}{|c|}{ Healthy participants } & \multicolumn{5}{|c|}{ Patients with stroke } \\
\hline & \multicolumn{3}{|c|}{ Age } & \multicolumn{2}{|c|}{ Education } & \multicolumn{3}{|c|}{ Age } & \multicolumn{2}{|c|}{ Education } \\
\hline & $\begin{array}{c}<50 \\
(n=22)\end{array}$ & $\begin{array}{c}50-69 \\
(n=18)\end{array}$ & $\begin{array}{c}>69 \\
(n=20)\end{array}$ & $\begin{array}{c}\text { Secondary } \\
(n=20)\end{array}$ & $\begin{array}{c}\text { Tertiary } \\
(n=40)\end{array}$ & $\begin{array}{c}<50 \\
(n=45)\end{array}$ & $\begin{array}{c}50-69 \\
(n=85) \\
\end{array}$ & $\begin{array}{c}>69 \\
(n=75)\end{array}$ & $\begin{array}{c}\text { Secondary } \\
(n=64)\end{array}$ & $\begin{array}{c}\text { Tertiary } \\
(n=141)\end{array}$ \\
\hline Picture Naming/Accuracy & 3 & 4 & 2 & 2 & 3 & 0 & 0 & 1 & 0 & 0 \\
\hline Picture Pointing/Accuracy & 3 & 3 & 3 & 3 & 3 & 2 & 0 & 2 & 1 & 2 \\
\hline Orientation/Accuracy & 4 & 4 & 2 & 2 & 3 & 2 & 0 & 1 & 1 & 1 \\
\hline Visual Field/Accuracy & 4 & 4 & 2 & 4 & 3 & 2 & 2 & 2 & 3 & 2 \\
\hline Sentence Reading/Accuracy & 15 & 15 & 15 & 15 & 15 & 0 & 0 & 2 & 4 & 4 \\
\hline Number Writing/Accuracy & 3 & 3 & 2 & 3 & 3 & 0 & 0 & 1 & 0 & 0 \\
\hline Calculation/Accuracy & 3 & 3 & 2 & 2 & 3 & 2 & 1 & 1 & 1 & 1 \\
\hline Broken Hearts/Accuracy & 38 & 42 & 0 & 38 & 44 & 13 & 0 & 0 & 0 & 3 \\
\hline Broken Hearts/Space Asymmetry & -4 & -3 & -4 & -4 & -3 & -7 & -9 & -10 & -7 & -9 \\
\hline Gesture Imitation/Accuracy & 11 & 9 & 4 & 4 & 7 & 2 & 0 & 1 & 1 & 1 \\
\hline Verbal Recall/Accuracy & 1 & 0 & 0 & 0 & 0 & 0 & 0 & 0 & 0 & 0 \\
\hline Verbal Recognition/Accuracy & 3 & 3 & 0 & 0 & 1 & 0 & 0 & 0 & 0 & 0 \\
\hline Episodic Recognition/Accuracy & 4 & 4 & 1 & 2 & 3 & 1 & 1 & 1 & 1 & 1 \\
\hline Mixed Executive Task/Accuracy & 7 & 11 & 1 & 3 & 6 & 0 & 0 & 0 & 0 & 1 \\
\hline Executive Score/Accuracy & -1 & -2 & -3 & -3 & -3 & -2 & -2 & -2 & -1 & -1 \\
\hline
\end{tabular}

Note. Rus-OCS $=$ Russian version of the Oxford Cognitive Screen.

\section{Reliability}

Results of test-retest reliability analyses for healthy and clinical groups are shown in Table 5. Retest data were obtained only for Version A because of the small sample, so caution is needed when using Version B. There were no significant differences between test and retest scores for any subtask. Correlation coefficients were established at the $p<.001$ level for all subtasks for healthy group and at least at the $p<.05$ level for stroke patients. Thus, we demonstrated a fair level of test-retest reliability for Rus-OCS Version A.

\section{Convergent Validity, Sensitivity, and Specificity}

Table 6 shows results of tests for convergent validity, sensitivity, and specificity of Rus-OCS subtasks. The correlation coefficients varied from a moderate level $\left(r_{\mathrm{p}}=.32-.49\right)$ for Picture Naming and Pointing, Sentence Reading, Number Writing, Calculation, Verbal and Episodic Recognition, and the Mixed Executive function task to a high level $\left(r_{\mathrm{p}}=.50-1\right)$ for the subtasks of Orientation, Visual Field, Broken Hearts accuracy and asymmetry, Gesture Imitation, Verbal Recall, and Executive score. The range of sensitivity of the Rus-OCS varied from .56 to $1(M=.79, S D=$ $.09)$. The lowest sensitivity value (.56) was observed for Episodic Recognition. Possible reasons for these results are discussed below. Specificity varied from .73 to $1(M=.86, S D=.12)$.

\section{Differences Between Clinical Subgroups}

As predicted, we found that Rus-OCS subtasks differentiated clinical groups with differential lesion location: left-hemisphere (LH), right-hemisphere (RH), and bilateral (BL) lesions (see Table 7). Chi-square values found significant differences on language tasks (i.e., Picture Naming and Pointing, Sentence Reading, Verbal Recall) and visual attention tasks (i.e., Broken Hearts accuracy and asymmetry). LH group had significantly lower scores on Picture
Naming $\left(\chi^{2}=3.93\right)$ in comparison with the BL group. On the Picture Pointing task, LH performed worse than RH group $\left(\chi^{2}=\right.$ 5.92). The LH group also had lower scores on the Sentence Reading subtask in comparison with $\mathrm{RH}\left(\chi^{2}=6.00\right)$ and $\mathrm{BL}\left(\chi^{2}=\right.$ 18.66). On the Verbal Recall subtask, LH was worse than the RH group $\left(\chi^{2}=8.54\right)$, and the BL group was better than $\mathrm{RH}\left(\chi^{2}=\right.$ 4.32). In sum, $\mathrm{LH}$ patients demonstrated lower performance on all language subtasks. Analysis of the Broken Hearts subtask scores measuring visual neglect and selective attention identified that $\mathrm{RH}$ patients performed worse than LH in the accuracy subtask $\left(\chi^{2}=\right.$ $7.51)$ and worse than BL on asymmetry measures $\left(\chi^{2}=5.34\right)$. We analyzed impairment ratios based on 5th and 95th percentiles derived from the healthy sample for each pair of clinical groups with different lesion location. The null hypothesis is that proportions of impaired and unimpaired results should be equal. We can reject this hypothesis as there were significant differences in impairment ratios on tests of language processing: Picture Naming $\left(\chi^{2}=8.91\right)$, Pointing $\left(\chi^{2}=7.03\right)$, Sentence Reading $\left(\chi^{2}=5.01\right)$, Verbal Recall $\left(\chi^{2}=9.43\right)$, Recognition $\left(\chi^{2}=3.98\right)$, and visuospatial processing (Space Asymmetry; $\chi^{2}=4.89$ ) for the groups with unilateral left or right hemisphere damage. Furthermore, in comparison to the BL group, LH patients had a significantly greater incidence of impairment on verbal tasks: Sentence Reading $\left(\chi^{2}=16.06\right)$, Verbal Recall $\left(\chi^{2}=4.50\right)$, Calculation $\left(\chi^{2}=4.72\right)$, and Episodic Recognition $\left(\chi^{2}=10.05\right)$. In comparison to $\mathrm{BL}$ group, $\mathrm{RH}$ patients had a significantly greater incidence of impairment on Space Asymmetry subtask $\left(\chi^{2}=8.44\right)$ while being intact on the Picture Pointing subtask $\left(\chi^{2}=4.18\right)$.

\section{Discussion}

The results confirmed our expectation that the Rus-OCS would discriminate Russian speakers with stroke from healthy people. We also found that patients with left hemisphere lesions (LH) perform worse on subtasks that test language and verbal functions 
Table 5

Descriptive Statistics and Comparison of Test and Retest Performance (After 3 to 7 Days) for 42 Healthy Participants and for 15 Stroke Patients

\begin{tabular}{|c|c|c|c|c|c|c|c|}
\hline \multirow[b]{2}{*}{ Rus-OCS task/Measure } & \multicolumn{2}{|c|}{ First test } & \multicolumn{2}{|c|}{ Second test } & \multirow{2}{*}{$\begin{array}{c}\begin{array}{c}\text { Wilcoxon signed } \\
\text { rank test }\end{array} \\
P\end{array}$} & \multirow{2}{*}{$\begin{array}{c}\text { Exact score of } \\
\text { agreement }\end{array}$} & \multirow[b]{2}{*}{$r_{\mathrm{p}}$} \\
\hline & $M$ & $S D$ & $M$ & $S D$ & & & \\
\hline & \multicolumn{7}{|c|}{ Healthy patients $(n=45)$} \\
\hline Picture Naming/Accuracy & 3.83 & .49 & 3.90 & .29 & .18 & 95 & $.73^{* *}$ \\
\hline Picture Pointing/Accuracy & 3 & 0 & 3 & 0 & 1 & 100 & - \\
\hline Orientation/Accuracy & 3.98 & .15 & 3.98 & .16 & 1 & 100 & $1^{* * *}$ \\
\hline Visual Field/Accuracy & 3.95 & .30 & 3.95 & .31 & 1 & 100 & $1^{* * *}$ \\
\hline Sentence Reading/Accuracy & 15 & 0 & 15 & 0 & 1 & 100 & - \\
\hline Number Writing/Accuracy & 2.98 & .154 & 3 & 0 & .32 & 98 & - \\
\hline Calculation/Accuracy & 3.69 & .46 & 3.81 & .40 & .59 & 83 & $.59^{* *}$ \\
\hline Broken Hearts/Accuracy & 45.19 & 7.734 & 45.36 & 7.57 & .22 & 79 & $.80^{* *}$ \\
\hline Broken Hearts/Space Asymmetry & -.11 & 1.02 & -.10 & 1.05 & .82 & 90 & $.73^{* *}$ \\
\hline Gesture Imitation/Accuracy & 11 & 1.41 & 11.05 & 1.45 & .53 & 83 & $.94^{* *}$ \\
\hline Verbal Recall/Accuracy & 2.57 & 1.346 & 2.74 & 1.11 & .12 & 71 & $.86^{* *}$ \\
\hline Verbal Recognition/Accuracy & 3.64 & .821 & 3.74 & .70 & .16 & 88 & $.85^{* *}$ \\
\hline Episodic Recognition/Accuracy & 3.86 & .354 & 3.90 & .30 & .32 & 90 & $.56^{* *}$ \\
\hline Mixed Executive task/Accuracy & 12.07 & 2.37 & 12.12 & 2.37 & 1 & 88 & $.97^{* *}$ \\
\hline \multirow[t]{2}{*}{ Executive score/Accuracy } & -.21 & 2.18 & -.18 & 2.23 & .58 & 100 & $1^{* * *}$ \\
\hline & \multicolumn{7}{|c|}{ Patients with stroke $(n=15)$} \\
\hline Picture Naming/Accuracy & 3.22 & .81 & 3.66 & .68 & .33 & 40 & $.60^{*}$ \\
\hline Picture Pointing/Accuracy & 2.83 & .38 & 2.66 & .69 & 1 & 72 & $1^{* * *}$ \\
\hline Orientation/Accuracy & 3.71 & .47 & 3.33 & 1.71 & 1 & 61 & $.49^{*}$ \\
\hline Visual Field/Accuracy & 3.78 & .65 & 3.71 & .83 & .95 & 100 & $1^{* * *}$ \\
\hline Sentence Reading/Accuracy & 11.11 & 5.35 & 11.39 & 3.60 & .56 & 40 & $.59^{* *}$ \\
\hline Number Writing/Accuracy & 2.61 & .85 & 2.61 & .78 & 1 & 93 & $.74^{* *}$ \\
\hline Calculation/Accuracy & 3.0 & 1.28 & 3.17 & 1.30 & .83 & 83 & $.96^{* *}$ \\
\hline Broken Hearts/Accuracy & 29.83 & 12.93 & 30.04 & 13.12 & .22 & 41 & $.54^{*}$ \\
\hline Broken Hearts/Space Asymmetry & -2.72 & 3.61 & -3.01 & 4.54 & .28 & 44 & $.48^{*}$ \\
\hline Gesture Imitation/Accuracy & 8.38 & 3.05 & 8.11 & 3.79 & .40 & 56 & $.83^{* *}$ \\
\hline Verbal Recall/Accuracy & .61 & .92 & .70 & 1.07 & .15 & 44 & $.49^{*}$ \\
\hline Verbal Recognition/Accuracy & 1.89 & 1.28 & 1.92 & 1.34 & .89 & 72 & $.66^{* *}$ \\
\hline Episodic Recognition/Accuracy & 2.56 & .98 & 2.50 & 1.25 & .59 & 67 & $.58^{*}$ \\
\hline Mixed Executive task/Accuracy & 6.72 & 4.60 & 7.03 & 3.67 & .81 & 83 & $.73^{*}$ \\
\hline Executive score/Accuracy & 1.28 & 1.96 & 1.27 & 1.46 & 1 & 100 & $1^{* * *}$ \\
\hline
\end{tabular}

Note. Dashes indicate that we could not compute the Pearson correlation because at least one group's scores were constant.

${ }^{*} p<.05$. *** $p<.001$.

whereas patients with right hemisphere lesions (RH) perform worse on subtasks that tests visuospatial functions. Most critically, Rus-OCS yielded reliable and valid responses and acceptable psychometric properties. We contend that the translation and adaptation of cultural and linguistic properties of the OCS for Russian speakers is reasonable given the similarities with normative scores of the original U.K. version of OCS and other studies (Kong et al., 2017; Hong et al., 2018).

All subtasks in Rus-OCS retained their purpose despite translation. In the Sentence Reading subtask, a new sentence was created to provide an equivalent level of complexity to the OCS for Russian speakers. The OCS Sentence Reading subtask is composed of four target words which require lexical knowledge for correct oral reading. The data for the 5 th percentiles in the memory subtasks requiring the retrieval of words was equivalent to items in the OCS, so the same level of difficulty can be assumed. The ceiling effect for healthy sample in the Sentence Reading task can be due to more automatized and rapid reading explained by relatively high level of orthographic transparency in Russian (see Ulicheva et al., 2016). A majority of participants in the LH group with aphasia performed significantly worse than the healthy group supporting the conclusion that the Sentence Reading subtask discriminates patients with language deficits.

We noted during testing that most unimpaired participants aged 66 years and older named the picture flamingo, although two alternative names produced were heron and stork. This is possibly because the post-World War II Soviet generation (after 1950 year of birth) had limited access to higher education, so their lack of general knowledge might be a contributing factor to the present results. In addition, the typical age of retirement in Russia is 55 years for women and 60 years for men (Kolev \& Pascal, 2002), potentially restricting familiarity with specific linguistic terms. The use of alternative correct answers in fact corresponds with the original OCS protocol where multiple options are correct for three items of the Picture Naming subtask of the Version A. For example, the first three pictures from the Picture Naming (Version A) could be named as (1) hippo or hippopotamus, (2) melon or watermelon, and (3) filing cabinet or chest of drawers. Thus, name agreement was considered as a necessary constraint by the OCS developers and they argued that this is as a part of cognitive load 
Table 6

Convergent Validity, Sensitivity, and Specificity of the Rus-OCS Tasks on Patients With Stroke

\begin{tabular}{|c|c|c|c|c|c|}
\hline Rus-OCS task/Measure & External task & $n$ & $r_{\mathrm{p}}$ & Sensitivity & Specificity \\
\hline Picture Naming/Accuracy & MoCA Naming & 127 & $.35^{* *}$ & .78 & .90 \\
\hline Picture Pointing/Accuracy & MoCA Naming & 127 & $.33^{* *}$ & .78 & .96 \\
\hline Orientation/Accuracy & MoCA Orientation & 127 & $.70^{* *}$ & .87 & .87 \\
\hline Visual Field/Accuracy & SCT Asymmetry & 50 & $.95^{* *}$ & .77 & .83 \\
\hline Sentence Reading/Accuracy & MoCA Sentence Repetition & 127 & $.33^{* * *}$ & .81 & .96 \\
\hline Number Writing/Accuracy & MoCA Clock Total & 127 & $.37^{* *}$ & .76 & .74 \\
\hline Calculation/Accuracy & MoCA Serial Subtraction & 127 & $.47^{* *}$ & .83 & .72 \\
\hline Broken Hearts/Accuracy & SCT Total & 50 & $.70^{* * *}$ & 1 & .73 \\
\hline Broken Hearts/Space Asymmetry & SCT Asymmetry & 50 & $.93^{* *}$ & .77 & .77 \\
\hline Gesture Imitation/Accuracy & Dynamic Kinesthetic Praxis & 127 & $.65^{* *}$ & .85 & .94 \\
\hline Verbal Recall/Accuracy & MoCA Delayed Recall & 127 & $.53^{* *}$ & .89 & .73 \\
\hline Verbal Recognition/Accuracy & MoCA Delayed Recall & 127 & $.45^{* *}$ & .80 & .90 \\
\hline Episodic Recognition/Accuracy & MoCA Delayed Recall & 127 & $.43^{* * *}$ & .56 & 1 \\
\hline Mixed Executive task/Accuracy & MoCA Trails & 127 & $.32^{* *}$ & .77 & .75 \\
\hline Executive score/Accuracy & MoCA Trails & 127 & $.54^{* *}$ & .87 & .91 \\
\hline
\end{tabular}

Note. Rus-OCS $=$ Russian version of the Oxford Cognitive Screen; MoCA = Montreal Cognitive Assessment; SCT = Star Cancellation Test. *** $p<.001$

of the picture naming subtask. Instead of flamingo, we recommended the item spanner (гаечный ключ/gaechnyi klyuch in Russian) from Version B, which was recognized by all participants and consists of two Russian words as the original item filing cabinet or chest of drawers.

We used Cronbach's alpha coefficients to test the internal reliability of Rus-OCS and values were at least .77, which is a high level of internal consistency (Tavakol \& Dennick, 2011). The HK-OCS study reported only one compound value of internal consistency equals to .725 (Kong et al., 2016). In contrast, for the OCS-P there were relatively low (.30 to .52) alpha coefficients for attention, memory, and language domains (Hong et al., 2018). We therefore submit that Rus-OCS has acceptable reliability comparable to other language versions.

Our goal was to provide normative data to be used as guidelines for clinical use of Rus-OCS. The current dataset yields a modest set of normative scores which may be used to estimate cognitive abilities in neuropsychological practice as well as for refinement of cut-offs on larger samples in future. The minimal differences with the OCS cutoffs (Demeyere et al., 2015) observed in the Broken Hearts Asymmetry scale and in the accuracy scores on the Executive function task are likely due to sampling differences and are not clinically significant given the range of scores in both tests Patients with clear problems in these domains would fall under the cut-off point in both cases. Also, the U.K. healthy group comprised 140 people, whereas only 60 healthy participants represented the Russian sample. Clearly, additional healthy participants' data is needed for the Rus-OCS cutoffs interpretation in future studies.

Normative scores were calculated for three different age groups and showed some differences on all subtasks except Picture Pointing and Sentence Reading, which demonstrated ceiling effects. Correlation analyses showed stronger effects of age on tests of

Table 7

Incidence of Impairments in Different Lesion Localizations

\begin{tabular}{|c|c|c|c|c|c|c|c|c|c|c|c|c|}
\hline \multirow[b]{2}{*}{ Rus-OCS task/Measure } & \multicolumn{3}{|c|}{$\begin{array}{l}\text { LH patients } \\
\quad(n=41)\end{array}$} & \multicolumn{3}{|c|}{$\begin{array}{l}\text { RH patients } \\
\quad(n=46)\end{array}$} & \multicolumn{3}{|c|}{$\begin{array}{l}\text { BL patients } \\
(n=118)\end{array}$} & \multicolumn{3}{|c|}{$\begin{array}{l}\chi^{2} \text { for the group scores/ } \\
\text { proportions of impairments }\end{array}$} \\
\hline & $M$ & $S D$ & $\%$ & $M$ & $S D$ & $\%$ & $M$ & $S D$ & $\%$ & LH-RH & LH-BL & RH-BL \\
\hline Picture Naming/Accuracy & 2.56 & 1.67 & 37 & 3.37 & .88 & 11 & 3.20 & 1.18 & 22 & $3.30 / 8.91^{* *}$ & $3.93^{*} / 3.37$ & $.39 / 2.69$ \\
\hline Picture Pointing/Accuracy & 2.75 & .62 & 20 & 3.00 & .37 & 2 & 2.86 & .47 & 13 & $5.92^{*} / 7.03^{* *}$ & $1.16 / 1.14$ & $4.90^{*} / 4.18^{*}$ \\
\hline Orientation/Accuracy & 3.39 & .95 & 39 & 3.46 & .94 & 33 & 3.54 & .89 & 29 & $.26 / .39$ & $1.86 / 1.47$ & $.47 / .23$ \\
\hline Visual Field/Accuracy & 3.84 & .49 & 17 & 3.6 & .88 & 22 & 3.69 & .78 & 19 & $1.92 / .30$ & $.95 / .16$ & $.51 / .10$ \\
\hline Sentence Reading/Accuracy & 9.71 & 6.49 & 61 & 12.30 & 4.72 & 37 & 13.16 & 4.37 & 26 & $6.0^{*} / 5.01^{*}$ & $18.66^{* *} / 16.06^{* *}$ & $2.04 / 1.83$ \\
\hline Number Writing/Accuracy & 2.27 & 1.15 & 59 & 2.74 & .71 & 50 & 2.38 & 1.02 & 50 & $3.01 / .63$ & $.07 / .89$ & $3.35 / .00$ \\
\hline Calculation/Accuracy & 2.92 & 1.29 & 59 & 3.44 & .89 & 48 & 3.18 & 1.04 & 39 & $1.95 / 1.0$ & $.40 / 4.72^{*}$ & $1.76 / 1.10$ \\
\hline Broken Hearts/Accuracy & 39.00 & 11.72 & 44 & 30.8 & 15.51 & 63 & 35.65 & 13.13 & 51 & $7.51^{* * *} / 3.2$ & $3.00 / .59$ & $3.24 / 1.98$ \\
\hline Broken Hearts/Space Asymmetry & 2.63 & 3.26 & 27 & 4.87 & 5.90 & 50 & 2.70 & 3.74 & 26 & $3.28 / 4.89^{*}$ & $3.57 / .01$ & $5.34^{*} / 8.44^{* * *}$ \\
\hline Gesture Imitation/Accuracy & 7.75 & 4.07 & 41 & 9.15 & 3.18 & 24 & 8.78 & 3.46 & 31 & $2.52 / 3.10$ & $1.71 / 1.39$ & $.29 / .89$ \\
\hline Verbal Recall/Accuracy & .43 & 1.08 & 95 & 1.48 & 1.37 & 70 & .91 & 1.33 & 81 & $8.54^{* *} / 9.43^{* *}$ & $3.31 / 4.50^{*}$ & $4.32 * / 2.69$ \\
\hline Verbal Recognition/Accuracy & 1.67 & 1.77 & 80 & 2.59 & 1.25 & 61 & 2.22 & 1.37 & 67 & $2.72 / 3.98^{*}$ & $2.20 / 2.68$ & $1.61 / .54$ \\
\hline Episodic Recognition/Accuracy & 2.76 & 1.45 & 66 & 3.51 & .80 & 48 & 3.28 & .99 & 37 & $3.86 / 2.86$ & $2.21 / 10.05^{* *}$ & $1.82 / 1.53$ \\
\hline Mixed Executive task/Accuracy & 8.71 & 3.94 & 56 & 7.46 & 4.59 & 61 & 8.67 & 4.80 & 47 & $.78 / .20$ & $.12 / 1.10$ & $2.47 / 2.69$ \\
\hline Executive score/Accuracy & 2.11 & 3.89 & 24 & 1.91 & 3.30 & 13 & -1.70 & 9.70 & 14 & $.56 / 1.86$ & $.19 / 2.15$ & $.20 / .05$ \\
\hline
\end{tabular}

Note. $\mathrm{LH}=$ left-hemisphere lesion; $\mathrm{RH}=$ right-hemisphere lesion; $\mathrm{BL}=$ bilateral lesions; $\%=$ percentage of impairments.

${ }^{*} p<.05 .{ }^{* *} p<.001$. 
memory, executive attention, and praxis. The results are consistent with findings in the Cantonese HK-OCS (Kong et al., 2016), although in that study higher scores were observed on number writing and sentence reading subtasks in a younger group. This difference could be explained by greater cognitive demands imposed when processing Chinese characters as confirmed by neural network modeling (Chang, Plaut, \& Perfetti, 2016).

An unexpected finding was that years of education were not significantly correlated with Rus-OCS scores. This contrasts with results from an Italian sample (Mancuso et al., 2016) and studies of MMSE and MoCA. For instance, studies of the MMSE use scoring adjustments for level of education (Crum, Anthony, Bassett, \& Folstein, 1993). Ardila, Ostrosky-Solis, Rosselli, and Gómez (2000) reported that cognitive decline is affected by education level. In the Italian study age and education independently predicted OCS performance. According to the results (Mancuso et al., 2016), level of education had an effect on all cognitive domains, but age influenced orientation only. The authors concluded that demographic factors constrained the use of OCS, and we acknowledge this requirement for future studies on a larger Russianspeaking sample. In our view, the low impact of education on performance reflects the potential of the OCS for use across a broad range of participants. Although the present results confirm the validity of Rus-OCS, it should be noted that all participants were recruited from the Moscow region. Residents of the Moscow region are more likely to be educated than residents from other parts of Russia because of greater access to media and higher mean income (Vishnevsky \& Bobylev, 2009). Therefore, before we can argue that the OCS is education-neutral, data from a wider range of Russian speakers is required. Our expectation however would be that level of education may have an effect on performance for Russian speakers residing outside of Moscow. Our results allow the conclusion that age exerts a wider influence on Rus-OCS scores than level of education, although this statement should be verified with a larger stratified sample too.

In the present study, test-retest reliability was established for all subtasks of the Rus-OCS on a healthy sample and a small group of 15 stroke patients. The next step in validation of the Rus-OCS must include assessment of test-retest reliability on a larger group of patients. For valid retest data collection, the choice of general clinic with newly admitted acute stroke patients receiving pharmacological intervention is preferable. We also acknowledge that inter- and intrarater reliability data must be added to meet criteria for the research use of the Rus-OCS.

We investigated the convergent validity of Rus-OCS based on its correlation with well-known and validated neuropsychological screens. All subtasks showed significant $(p<.05)$ correlations with comparable tasks. Moderate correlations were found on language, numerical, memory and switching subtasks which could be explained by slightly different nature of the comparable validation tasks taken from MoCA scale. For instance, the Rus-OCS Number Writing subtask was correlated with the MoCA Clock Drawing subtask because it includes writing numbers. We argue that the Clock Drawing subtask imposes more demands on the visuospatial system especially regarding the positioning of the clock hands. Although, in terms of the ecological validity of the subtask, the ability to write numbers as measured in the Rus-OCS looks more relevant to life demands for stroke survivors.
The choice of the use of MoCA subtasks for correlation with the Rus-OCS subtasks as a part of the convergent validity analysis was influenced by validation studies of the original OCS and HK-OCS. Similarly, the OCS-P used the Chinese Beijing version (MoCAChiB) as an external measurement of the criterion validity (Hong et al., 2018). However, the short subtasks of the MoCA often have very little range as they are normally interpreted as a part of the MoCA total score. This may lead to relatively small correlations with OCS subtasks. For example, MoCA Trail Making subtask was correlated with Rus-OCS Executive subtask while having only two values, zero and one. In future studies, the use of fully established and standardized tests for each cognitive domain separately is recommended for a more precise validation procedure.

Estimates of the diagnostic accuracy of Rus-OCS using measures of sensitivity and specificity were based on 5th and 95th percentiles derived from the healthy sample and compared with MoCA, SCT and LNB cutoffs taken from their official websites. The true negative rates (i.e., the percentage of participants who are correctly identified as not having impairment) revealed an acceptable to high level of sensitivity for all subtasks (range $=.73-1$ ), whereas the probability of impairment detection was again sufficient to high for all subtasks (range $=.76-1$ ), except for the episodic recognition subtask (.56). We suggest that lack of sensitivity may result from greater inclusivity of the Rus-OCS subtask in comparison with matching tasks for validation. For example, the Episodic Recognition subtask has a visual multiple-choice response mode which allows patients who have language difficulties or endotracheal tube to respond by pointing not speaking. Comparable scores on the test of episodic memory on the MoCA may be confounded, as a failure on this task may be due to inability to respond verbally. This argument is confirmed by the fact that, according to medical records, at least $20 \%(n=42)$ of the stroke sample were diagnosed with aphasia.

Our preliminary results confirmed that Rus-OCS can differentiate performance of cognitively impaired and unimpaired Russian speakers. Moreover, the Rus-OCS is sensitive to lesion lateralization in the case of left hemisphere brain damage with language subtasks (Picture Naming and Pointing, Sentence Reading, Verbal Recall) and right hemisphere brain damage on tests of visual attention (Broken Hearts Accuracy and Asymmetry). Our findings therefore confirm that the stroke patients with left hemisphere damage are likely to have impairment to language functions (object naming, oral reading, semantic processing, verbal recall). In contrast, stroke patients with right-hemisphere damage perform significantly worse on visuospatial attention tasks. Analysis of the impairment ratios within each group confirmed these results. We also compared results of the lesion groups with BL patients without clear lateralization of brain damage. The BL would be expected to show functional impairments typical of brain damage for either hemisphere but not show isolated impairment to language or visuospatial processing. The BL group thus serves as a control group for comparisons of specificity with $\mathrm{LH}$ and $\mathrm{RH}$ groups because all participants have brain damage that causes cognitive impairment. The comparisons between LH and BL groups and between $\mathrm{RH}$ and BL groups therefore allow conclusions about the specific impairments caused by damage to one hemisphere independent of the effects of brain damage per se. According to this reasoning, damage to the left hemisphere in the present sample leads to impairment in verbal functions whereas damage to the 
right hemisphere leads to impairment in visuospatial functions. These findings are compatible with what is known about the lateralization of cognitive functions in the brain. Moreover, in the HK-OCS validation study similar results were presented after paired-sample $t$ test between scores of left and right hemispheric stroke groups. According to Kong and colleagues (2016), significant differences between LH and RH groups were observed in the Sentence Reading, Orientation (free response), Verbal Memory (Recall), Number Writing, and Calculation subtasks for Cantonese speaking participants.

We seek to confirm the discriminative power of Rus-OCS for different lesion localizations in future studies. In the present study, the BL group had the largest number of participants and can be characterized as chronic (recurring strokes were noted in the records). This group had more diverse pathology with multifocal strokes but had been admitted to hospital with acute lesions during the data collection period. Not surprisingly, this group was characterized by diverse and widespread cognitive impairment in comparison to unilateral stroke patients and by some measures may be seen to have greater functional impairment due to extensive brain damage. We noted however, that BL stroke patients had better language performance than the LH as well as better visuospatial functioning in comparison with $\mathrm{RH}$ group. On the basis of the percentage of impairments for the BL group, we can conclude that memory (verbal and nonverbal), visual attention, and numerical function are vulnerable in Russian-speaking stroke patients with bilateral lesions. For the LH group, we found that calculation and episodic memory impairments are more likely when compared to patients with $\mathrm{BL}$ damage in addition to expected impairments to oral reading and verbal memory recall functions. For the RH group, we found that space asymmetry is more likely to be impaired when compared to patients with BL damage.

The lesion results described in the preceding text are impressionistic but consistent with results of previous studies (Kleinman et al., 2007; Kong et al., 2016; Xing et al., 2016). MRI studies show that language processing is left lateralized (Frost et al., 1999). Human neuropsychological research shows that LH patients have more impairments with calculation in comparison with $\mathrm{RH}$ patients (Rosselli \& Ardila, 1989). A higher percentage of failures on the episodic memory task in the LH group might be caused by the disturbed encoding processes associated with activity of left prefrontal cortex (Fletcher, Frith, \& Rugg, 1997). We would caution against direct comparisons between the LH and RH groups because cohort studies suggest that patients with RH lesions have fewer functional impairments initially and have a better cognitive recovery (Hochstenbach, den Otter, \& Mulder, 2003; Patel, Coshall, Rudd, \& Wolfe, 2003). One reason for this may be preservation of language abilities following damage to the right hemisphere. We contend that isolated damage to the right hemisphere results in fewer impairments compared with damage to the left hemisphere. It is not clear however if this is because of preserved language abilities only following brain damage. The RH group had no impairment to language, but Rus-OCS is designed to be insensitive to language impairment. Thus, we expected performance to dissociate impairments to cognitive processes that do not depend on language or visuospatial processing ability. We therefore conclude that the differential impairments observed for patients with left and right lateralized brain damage are resilient to adaptations that diminish language and visuospatial processing abilities.

The results with lateralized deficits might help specialists with the selection of lesion-laterality specific treatment methods. However, more detailed investigation of patients with the bilateral stroke is required because of the increasing incidence of chronic stroke in Russia (Gusev, Skvortsova, \& Stakhovskaia, 2002; Mukherjee \& Patil, 2011). Presentation of bilateral stroke in the AQ: 9 Russian health care system is often accompanied by diagnosis of discirculatory encephalopathy (Levin, 2012). This is a term used in Eastern Europe to describe the chronic progressive form of cerebrovascular pathology leading to multifocal or diffuse brain lesions and causing a variety of neurological and neuropsychological disorders (Yakhno, Levin, \& Damulin, 2001). The definition is similar to the concept of small vessel disease (SVD) in Western countries (Pantoni, Poggesi, \& Inzitari, 2009; Pantoni, 2010). It is notable that the literature on the clinical features of SVD has not made an impact in Russia to date. As the Rus-OCS has good sensitivity, we suggest it could be used at the earliest stage of progressive vascular disease.

\section{Limitations}

The limitations of this study lie in the normative sample size and type and gender imbalance, thus preventing generalization of the results to the Russian population. Another serious flaw of the present work is lack of test-retest, inter- and intrarater reliability coefficients. Test-retest reliability should be established using a larger clinical sample and using Version B of the Rus-OCS as well, as it is important for clinicians to know whether the same patient will score similarly on the same test when no changes in underlying impairments can be expected. Because test-retest reliability could not be ascertained with the most of the clinical sample, this issue must to be addressed in future studies together with all other reliability parameters.

One more limitation is the lack of sensitivity of the Episodic Recognition caused by differentiating level of difficulty in the validation task. We note that language and visual impairments add noise to the data. In future studies, use of established and standardized tests for all cognitive domains separately is recommended for further exploration of the Rus-OCS convergent validity.

Another part of construct validity, divergent validity, was not reported in the present work. On the basis of results of the original OCS validation study (Demeyere et al., 2015), we would expect low correlation values of all OCS subtasks with Barthel Index measuring physical activities of daily life and quality of life. Additionally, low correlation values were reported between memory and attention/executive domains and between executive and praxis subtasks of the UK-OCS.

\section{Conclusions}

In sum, the Rus-OCS has a fair level of validity based on this preliminary study allowing us to recommend it for further validation and use in clinical practice with Russian speakers. During development, we received positive comments from members of the multidisciplinary clinical team in Moscow. The depiction of patient profiles was found to be very convenient for family members and caregivers, providing them with additional cues about the 
cognitive strengths and weaknesses of a patient. Because of its speed and easy administration, comprehensive assessment, and language neutrality, the Rus-OCS is suitable for inclusion into clinical practice and routine assessments immediately, with further development to follow.

\section{References}

Abutalebi, J., \& Weekes, B. S. (2014). The cognitive neurology of bilingualism in the age of globalization. Behavioural Neurology, 2014, Article ID 536727. http://dx.doi.org/10.1155/2014/536727

Akhutina, T. V. (2015). Luria's classification of aphasias and its theoretical basis. Aphasiology, 30, 878-897. http://dx.doi.org/10.1080/02687038 .2015 .1070950

Akhutina, T. V., \& Melikyan, Z. A. (2012). Neuropsychological assessment: An overview of modern tendencies (Dedicated to the 110th anniversary of A. R. Luria). Clinical Psychology and Special Education, 1(2). Retrieved from http://psyjournals.ru/en/psyclin/2012/n2/54529 .shtml

Ardila, A., Ostrosky-Solis, F., Rosselli, M., \& Gómez, C. (2000). Agerelated cognitive decline during normal aging: The complex effect of education. Archives of Clinical Neuropsychology, 15, 495-513.

Barber, M., \& Stott, D. J. (2004). Validity of the Telephone Interview for Cognitive Status (TICS) in post-stroke subjects. International Journal of Geriatric Psychiatry, 19, 75-79. http://dx.doi.org/10.1002/gps.1041

Barker-Collo, S., \& Feigin, V. (2006). The impact of neuropsychological deficits on functional stroke outcomes. Neuropsychology Review, 16, 53-64. http://dx.doi.org/10.1007/s11065-006-9007-5

Barnay, J. L., Wauquiez, G., Bonnin-Koang, H. Y., Anquetil, C., Pérennou, D., Piscicelli, C., . . Benaim, C. (2014). Feasibility of the cognitive assessment scale for stroke patients (CASP) vs. MMSE and MoCA in aphasic left hemispheric stroke patients. Annals of Physical and Rehabilitation Medicine, 57, 422-435. http://dx.doi.org/10.1016/j.rehab.2014 .05 .010

Bour, A., Rasquin, S., Boreas, A., Limburg, M., \& Verhey, F. (2010). How predictive is the MMSE for cognitive performance after stroke? Journal of Neurology, 257, 630-637. http://dx.doi.org/10.1007/s00415-0095387-9

Burgess, P. W., Alderman, N., Forbes, C., Costello, A., Coates, L. M., Dawson, D. R., . . . Channon, S. (2006). The case for the development and use of "ecologically valid" measures of executive function in experimental and clinical neuropsychology. Journal of the International Neuropsychological Society, 12, 194-209. http://dx.doi.org/10.1017/ S1355617706060310

Carod-Artal, J., Egido, J. A., González, J. L., \& Varela de Seijas, E. (2000). Quality of life among stroke survivors evaluated 1 year after stroke: Experience of a stroke unit. Stroke, 31, 2995-3000. http://dx.doi.org/10 .1161/01.STR.31.12.2995

Carson, A. J., MacHale, S., Allen, K., Lawrie, S. M., Dennis, M., House, A., \& Sharpe, M. (2000). Depression after stroke and lesion location: a systematic review. The Lancet, 356, 122-126. http://dx.doi.org/10.1016/ S0140-6736(00)02448-X

Chang, L. Y., Plaut, D. C., \& Perfetti, C. A. (2016). Visual complexity in orthographic learning: Modeling learning across writing system variations. Scientific Studies of Reading, 20, 64-85. http://dx.doi.org/10 $.1080 / 10888438.2015 .1104688$

Corbetta, M., Ramsey, L., Callejas, A., Baldassarre, A., Hacker, C. D., Siegel, J. S., . . . Shulman, G. L. (2015). Common behavioral clusters and subcortical anatomy in stroke. Neuron, 85, 927-941. http://dx.doi .org/10.1016/j.neuron.2015.02.027

Cronbach, L. J. (1951). Coefficient alpha and the internal structure of tests. psychometrika, 16, 297-334.

Crum, R. M., Anthony, J. C., Bassett, S. S., \& Folstein, M. F. (1993). Population-based norms for the Mini-Mental State Examination by age and educational level. Journal of the American Medical Association, 269, 2386-2391. http://dx.doi.org/10.1001/jama.1993.03500180078038

Cullen, B., O’Neill, B., Evans, J. J., Coen, R. F., \& Lawlor, B. A. (2007). A review of screening tests for cognitive impairment. Journal of Neurology, Neurosurgery, and Psychiatry, 78, 790-799. http://dx.doi.org/ 10.1136/jnnp.2006.095414

Cumming, T. B., Marshall, R. S., \& Lazar, R. M. (2013). Stroke, cognitive deficits, and rehabilitation: Still an incomplete picture. International Journal of Stroke, 8, 38-45. http://dx.doi.org/10.1111/j.1747-4949.2012 .00972.x

de Koning, I., van Kooten, F., Dippel, D. W., van Harskamp, F., Grobbee, D. E., Kluft, C., \& Koudstaal, P. J. (1998). The CAMCOG: A useful screening instrument for dementia in stroke patients. Stroke, 29, 2080 2086. http://dx.doi.org/10.1161/01.STR.29.10.2080

Delis, D. C., Kramer, J. H., Kaplan, E., \& Holdnack, J. (2004). Reliability and validity of the Delis-Kaplan Executive Function System: An update Journal of the International Neuropsychological Society, 10, 301-303. http://dx.doi.org/10.1017/S1355617704102191

Demeyere, N., Riddoch, M. J., Slavkova, E. D., Bickerton, W. L., \& Humphreys, G. W. (2015). The Oxford Cognitive Screen (OCS): Validation of a stroke-specific short cognitive screening tool. Psychological Assessment, 27, 883-894. http://dx.doi.org/10.1037/pas0000082

Demeyere, N., Riddoch, M. J., Slavkova, E. D., Jones, K., Reckless, I., Mathieson, P., \& Humphreys, G. W. (2016). Domain-specific versus generalized cognitive screening in acute stroke. Journal of Neurology, 263, 306-315. http://dx.doi.org/10.1007/s00415-015-7964-4

Dong, Y., Sharma, V. K., Chan, B. P. L., Venketasubramanian, N., Teoh, H. L., Seet, R. C. S., . . . Chen, C. (2010). The Montreal Cognitive Assessment (MoCA) is superior to the Mini-Mental State Examination (MMSE) for the detection of vascular cognitive impairment after acute stroke. Journal of the Neurological Sciences, 299, 15-18. http://dx.doi .org/10.1016/j.jns.2010.08.051

Easton, J. D., Saver, J. L., Albers, G. W., Alberts, M. J., Chaturvedi, S., Feldmann, E., . . Lutsep, H. L. (2009). Definition and evaluation of transient ischemic attack: a scientific statement for healthcare professionals from the American Heart Association/American Stroke Association Stroke Council; Council on Cardiovascular Surgery and Anesthesia; Council on Cardiovascular Radiology and Intervention; Council on Cardiovascular Nursing; and the Interdisciplinary Council on Peripheral Vascular Disease: the American Academy of Neurology affirms the value of this statement as an educational tool for neurologists. Stroke, 40, 2276-2293. http://dx.doi.org/10.1161/STROKEA

Egerházi, A., Berecz, R., Bartók, E., \& Degrell, I. (2007). Automated Neuropsychological Test Battery (CANTAB) in mild cognitive impairment and in Alzheimer's disease. Progress in Neuro-Psychopharmacology \& Biological Psychiatry, 31, 746-751. http://dx.doi.org/ 10.1016/j.pnpbp.2007.01.011

Fletcher, P. C., Frith, C. D., \& Rugg, M. D. (1997). The functional neuroanatomy of episodic memory. Trends in Neurosciences, 20, 213 218. http://dx.doi.org/10.1016/S0166-2236(96)01013-2

Friedman, P. J. (1992). The star cancellation test in acute stroke. Clinical Rehabilitation, 6, 23-30. http://dx.doi.org/10.1177/026921559200600104

Frost, J. A., Binder, J. R., Springer, J. A., Hammeke, T. A., Bellgowan, P. S., Rao, S. M., \& Cox, R. W. (1999). Language processing is strongly left lateralized in both sexes. Evidence from functional MRI. Brain: A Journal of Neurology, 122(Pt 2), 199-208. http://dx.doi.org/10.1093/ brain/122.2.199

Golden, C. J. (1980). The Luria-Nebraska neuropsychological battery: Manual. Torrance, CA: Western Psychological Services.

Gusev, E. I., Skvortsova, V. I., \& Stakhovskaia, L. V. (2002). Epidemiologiya insul'ta v Rossii [Epidemiology of stroke in Russia]. Zhurnal nevrologii i psikhiatrii imeni SS Korsakova, 8, 4-9.

Hachinski, V., Iadecola, C., Petersen, R. C., Breteler, M. M., Nyenhuis, D. L., Black, S. E., . . . Vinters, H. V. (2006). National Institute of 
Neurological Disorders and Stroke-Canadian stroke network vascular cognitive impairment harmonization standards. Stroke, 37, 2220-2241. http://dx.doi.org/10.1161/01.STR.0000237236.88823.47

Han, D. Y., Anderson, A. J., Jones, J. E., Hermann, B. P., \& Sattin, J. A. (2014). Neuropsychology in multidisciplinary stroke care: Clinical feasibility of the NINDS-CSN vascular cognitive impairment harmonization standards. International Scholarly Research Notices, 2014, Article ID 216024. http://dx.doi.org/10.1155/2014/216024

AQ: 10 Helm-Estabrooks, N. (2001). Cognitive linguistic quick test: CLQT. San Antonio, TX: Pearson/PsychCorp.

Hochstenbach, J. B., den Otter, R., \& Mulder, T. W. (2003). Cognitive recovery after stroke: A 2-year follow-up. Archives of Physical Medicine and Rehabilitation, 84, 1499-1504. http://dx.doi.org/10.1016/S00039993(03)00370-8

Hong, W. J., Tao, J., Wong, A. W. K., Yang, S. L., Leung, M. T., Lee, T. M. C., .. . Chen, L. D. (2018). Psychometric properties of the Chinese (Putonghua) version of the Oxford Cognitive Screen (OCS-P) in subacute poststroke patients without neglect. BioMed Research International, 2018, Article ID 6827854. http://dx.doi.org/10.1155/2018/ 6827854

Humphreys, G. W., Bickerton, W. L., Samson, D., \& Riddoch, M. J. (2012). The Birmingham Cognitive Screen (BCoS). London, UK: Psychology Press.

Humphreys, G. W., Duta, M. D., Montana, L., Demeyere, N., McCrory, C., Rohr, J., . . Berkman, L. (2017). Cognitive function in low-income and low-literacy settings: Validation of the tablet-based Oxford Cognitive Screen in health and aging in Africa: A longitudinal study of an INDEPTH community in South Africa (HAALSI). The Journals of Gerontology Series B, Psychological Sciences and Social Sciences, 72, 38-50. http://dx.doi.org/10.1093/geronb/gbw139

Ivanova, M., \& Hallowell, B. (2009). Short form of the Bilingual Aphasia Test in Russian: Psychometric data of persons with aphasia. Aphasiology, 23, 544-556. http://dx.doi.org/10.1080/02687030701800784

Kertesz, A. (2006). Western aphasia battery-revised (WAB-R): Examiner's

AQ: 11 manual. San Antonio, TX: PsychCorp/Harcort Assessment Incorporation.

Kleinman, J. T., Newhart, M., Davis, C., Heidler-Gary, J., Gottesman, R. F., \& Hillis, A. E. (2007). Right hemispatial neglect: Frequency and characterization following acute left hemisphere stroke. Brain and Cognition, 64, 50-59. http://dx.doi.org/10.1016/j.bandc.2006.10.005

Kolev, A., \& Pascal, A. (2002). What keeps pensioners at work in Russia? Evidence from Household Panel data. Retrieved from https:// onlinelibrary.wiley.com/doi/abs/10.1111/1468-0351.00102

Kong, A. P. H., Chan, J., Lau, J. K. L., Bickerton, W. L., Weekes, B., \& Humphreys, G. (2017). Developing a Cantonese version of Birmingham Cognitive Screen for stroke survivors in Hong Kong. Communication Disorders Quarterly, 39, 387-401. http://dx.doi.org/10.1177/ 1525740117720382

Kong, A. P. H., Lam, P. H. P., Ho, D. W. L., Lau, J. K., Humphreys, G. W., Riddoch, J., \& Weekes, B. (2016). The Hong Kong version of the Oxford Cognitive Screen (HK-OCS): Validation study for Cantonesespeaking chronic stroke survivors. Neuropsychology, Development, and Cognition Section B, Aging, Neuropsychology and Cognition, 23, 530548. http://dx.doi.org/10.1080/13825585.2015.1127321

Kornev, A. N., Rakhlin, N., \& Grigorenko, E. L. (2010). Dyslexia from a cross-linguistic and cross-cultural perspective: The case of Russian and Russia. Learning Disabilities (Weston, Mass.), 8, 41-69.

Kuzmina, E., Humphreys, G. W., Riddoch, M. J., Skvortsov, A. A., \& Weekes, B. S. (2018). Preliminary validation study of the Russian Birmingham Cognitive Screen. Journal of Clinical and Experimental Neuropsychology, 40, 1-16. http://dx.doi.org/10.1080/13803395.2017 .1301884
Lai, S. M., Studenski, S., Duncan, P. W., \& Perera, S. (2002). Persisting consequences of stroke measured by the Stroke Impact Scale. Stroke, 33, 1840-1844. http://dx.doi.org/10.1161/01.STR.0000019289.15440.F2

Lam, P., Kong, A., Ho, D., Humphreys, G., \& Weekes, B. (2014). Cantonese version of the Oxford Cognitive Screen (OCS): Validation for stroke survivors in Hong Kong. Frontiers in Psychology, 5. Psychol. Conference Abstract: Academy of Aphasia - 52nd Annual Meeting. http://dx.doi.org/10.3389/conf.fpsyg.2014.64.00005

Levin, O. S. (2012). Discirculatory encephalopathy: An anachronism or a clinical reality. Journal of Current Therapy in Psychiatry and Neurology, 3, 40-46.

Levin, O. S., Lavrov, A. Y., Lyashenko, E. A., Vasenina, E. E., Trusova, N. A., Datieva, V. K., . . Pilipenko, A. Y. (2015). Validation of the Russian version of the modified Addenbrooke's cognitive examination for Alzheimer's disease diagnosis. Zhurnal nevrologii i psikhiatrii imeni SS Korsakova, 115, 36-39.

Luria, A. R. (1966). The human brain and psychological processes. New York, NY: Harper \& Row.

Luria, A. R., \& Hutton, J. T. (1977). A modern assessment of the basic forms of aphasia. Brain and Language, 4, 129-151. http://dx.doi.org/ 10.1016/0093-934X(77)90012-8

Luria, A. R., \& Majovski, L. V. (1977). Basic approaches used in American and Soviet clinical neuropsychology. American Psychologist, 32, 959-968. http://dx.doi.org/10.1037/0003-066X.32.11.959

Makeeva, O., Markova, V., Zhukova, I., Melikyan, Z., Minaycheva, L., Buikin, S., \& Starinskaya, E. (2012). Montreal Cognitive Assessment (MoCA) population-based study of Russian elderly. Alzheimer's \& Dementia, 8(4), S773. http://dx.doi.org/10.1016/j.jalz.2013.08.108

Mancuso, M., Demeyere, N., Abbruzzese, L., Damora, A., Varalta, V., Pirrotta, F., . . . the Italian OCS Group. (2018). Using the Oxford Cognitive Screen to detect cognitive impairment in stroke patients: A comparison with the Mini-Mental State Examination. Frontiers in Neurology, 9, 101. http://dx.doi.org/10.3389/fneur.2018.00101

Mancuso, M., Varalta, V., Sardella, L., Capitani, D., Zoccolotti, P., Antonucci, G., . . . the Italian OCS Group. (2016). Italian normative data for a stroke specific cognitive screening tool: The Oxford Cognitive Screen (OCS). Neurological Sciences, 37, 1713-1721. http://dx.doi.org/10 .1007/s10072-016-2650-6

Maruff, P., Thomas, E., Cysique, L., Brew, B., Collie, A., Snyder, P., \& Pietrzak, R. H. (2009). Validity of the CogState brief battery: Relationship to standardized tests and sensitivity to cognitive impairment in mild traumatic brain injury, schizophrenia, and AIDS dementia complex. Archives of Clinical Neuropsychology, 24, 165-178. http://dx.doi.org/ 10.1093/arclin/acp010

Massa, M. S., Wang, N., Bickerton, W.-L., Demeyere, N., Riddoch, M. J., \& Humphreys, G. W. (2015). On the importance of cognitive profiling: A graphical modelling analysis of domain-specific and domain-general deficits after stroke. Cortex, 71, 190-204. http://dx.doi.org/10.1016/j .cortex.2015.06.006

McGovern, A., Pendlebury, S. T., Mishra, N. K., Fan, Y., \& Quinn, T. J. (2016). Test accuracy of informant-based cognitive screening tests for diagnosis of dementia and multidomain cognitive impairment in stroke. Stroke, 47, 329-335. http://dx.doi.org/10.1161/STROKEAHA.115 .011218

Moorhouse, P., \& Rockwood, K. (2008). Vascular cognitive impairment: Current concepts and clinical developments. Lancet Neurology, 7, $246-$ 255. http://dx.doi.org/10.1016/S1474-4422(08)70040-1

Morris, K., Hacker, V., \& Lincoln, N. B. (2012). The validity of the Addenbrooke's Cognitive Examination-Revised (ACE-R) in acute stroke. Disability and Rehabilitation, 34, 189-195. http://dx.doi.org/10 .3109/09638288.2011.591884

Mueller, J., Kiernan, R., \& Langston, J. W. (2011). Cognistat and Cognistat Assessment System manual. Fairfax, CA: Cognistat, Inc.
AQ: 12

(a)


Mukherjee, D., \& Patil, C. G. (2011). Epidemiology and the global burden of stroke. World neurosurgery, 76, S85-S90. http://dx.doi.org/10.1016/ j.wneu.2011.07.023

Mysiw, W. J., Beegan, J. G., \& Gatens, P. F. (1989). Prospective cognitive assessment of stroke patients before inpatient rehabilitation. The relationship of the Neurobehavioral Cognitive Status Examination to functional improvement. American Journal of Physical Medicine \& Rehabilitation, 68, 168-171. http://dx.doi.org/10.1097/ 00002060-198908000-00003

Nasreddine, Z. S., Phillips, N. A., Bédirian, V., Charbonneau, S., Whitehead, V., Collin, I., . . . Chertkow, H. (2005). The Montreal Cognitive Assessment, MoCA: A brief screening tool for mild cognitive impairment. Journal of the American Geriatrics Society, 53, 695-699. http:// dx.doi.org/10.1111/j.1532-5415.2005.53221.x

Nys, G. M. S., Van Zandvoort, M. J. E., De Kort, P. L. M., Jansen, B. P. W., Van der Worp, H. B., Kappelle, L. J., \& De Haan, E. H. F. (2005). Domain-specific cognitive recovery after first-ever stroke: A follow-up study of 111 cases. Journal of the International Neuropsychological Society, 11, 795-806. http://dx.doi.org/10.1017/ S1355617705050952

Oguro, H., Yamaguchi, S., Abe, S., Ishida, Y., Bokura, H., \& Kobayashi, S. (2006). Differentiating Alzheimer's disease from subcortical vascular dementia with the FAB test. Journal of Neurology, 253, 1490-1494. http://dx.doi.org/10.1007/s00415-006-0251-7

Panchenko, A., Loukachevitch, N., Ustalov, D., Paperno, D., Meyer, C., \& Konstantinova, N. (2018). RUSSE: The first workshop on Russian semantic similarity. Retrieved from https://arxiv.org/abs/1803.05820

Pantoni, L. (2010). Cerebral small vessel disease: From pathogenesis and clinical characteristics to therapeutic challenges. Lancet Neurology, 9, 689-701. http://dx.doi.org/10.1016/S1474-4422(10)70104-6

Pantoni, L., Poggesi, A., \& Inzitari, D. (2009). Cognitive decline and dementia related to cerebrovascular diseases: Some evidence and concepts. Cerebrovascular Diseases (Basel, Switzerland), 27(Suppl 1), 191-196. http://dx.doi.org/10.1159/000200459

Parsons, T. D. (2016). Clinical neuropsychology and technology. New York, NY: Springer Press. http://dx.doi.org/10.1007/978-3-319-31075-6

Patel, M. D., Coshall, C., Rudd, A. G., \& Wolfe, C. D. (2002). Cognitive impairment after stroke: Clinical determinants and its associations with long-term stroke outcomes. Journal of the American Geriatrics Society, 50, 700-706. http://dx.doi.org/10.1046/j.1532-5415.2002.50165.x

Patel, M., Coshall, C., Rudd, A. G., \& Wolfe, C. D. (2003). Natural history of cognitive impairment after stroke and factors associated with its recovery. Clinical Rehabilitation, 17, 158-166. http://dx.doi.org/10 $.1191 / 0269215503 \mathrm{cr} 5960 \mathrm{a}$

Pendlebury, S. T., Mariz, J., Bull, L., Mehta, Z., \& Rothwell, P. M. (2012). MoCA, ACE-R, and MMSE versus the National Institute of Neurological Disorders and Stroke-Canadian Stroke Network vascular cognitive impairment harmonization standards neuropsychological battery after tiA and stroke. Stroke, 43, 464-469. http://dx.doi.org/10.1161/ STROKEAHA.111.633586

Rasskazova, E. I., Kovyazina, M. S., \& Varako, N. A. (2016). Screening scales in neuropsychological rehabilitation: Opportunities, requirements and limitations. Bulletin of the South Ural State University, 9, 5-15. http://dx.doi.org/10.14529/psy160301

Raven, J. (2000). The Raven's progressive matrices: Change and stability over culture and time. Cognitive Psychology, 41, 1-48. http://dx.doi.org/ 10.1006/cogp.1999.0735

Richardson, M., Campbell, N., Allen, L., Meyer, M., \& Teasell, R. (2016). The stroke impact scale: Performance as a quality of life measure in a community-based stroke rehabilitation setting. Disability and Rehabilitation, 38, 1425-1430. http://dx.doi.org/10.3109/09638288.2015 .1102337
Robinson, G., \& Weekes, B. (2013). Rehabilitation in clinical neuropsychology. In G. Davey (Ed.), Clinical psychology: Topics in applied psychology (pp. 207-228). New York, NY: Routledge.

Rosselli, M., \& Ardila, A. (1989). Calculation deficits in patients with right and left hemisphere damage. Neuropsychologia, 27, 607-617. http://dx .doi.org/10.1016/0028-3932(89)90107-3

Stern, R. A., \& White, T. (2003). NAB, Neuropsychological Assessment Battery: Attention Module Stimulus Book. Form 2. Lutz, FL: Psychological Assessment Resources.

Tavakol, M., \& Dennick, R. (2011). Making sense of Cronbach's alpha. International Journal of Medical Education, 2, 53-55. http://dx.doi.org/ 10.5116/ijme.4dfb.8dfd

Thompson, H. S., \& Ryan, A. (2009). The impact of stroke consequences on spousal relationships from the perspective of the person with stroke. Journal of Clinical Nursing, 18, 1803-1811. http://dx.doi.org/10.1111/ j.1365-2702.2008.02694.x

Thompson, P. M., Stein, J. L., Medland, S. E., Hibar, D. P., Vasquez, A. A., Renteria, M. E., . . . the Alzheimer's Disease Neuroimaging Initiative, EPIGEN Consortium, IMAGEN Consortium, Saguenay Youth Study (SYS) Group. (2014). The ENIGMA Consortium: Largescale collaborative analyses of neuroimaging and genetic data. Brain Imaging and Behavior, 8, 153-182.

Ulicheva, A., Coltheart, M., Saunders, S., \& Perry, C. (2016). Phonotactic constraints: Implications for models of oral reading in Russian. Journal of Experimental Psychology: Learning, Memory, and Cognition, 42, 636-656. http://dx.doi.org/10.1037/xlm0000203

Vishnevsky, A. G., \& Bobylev, S. N. (2009). National human development report, Russian Federation, 2009. Russia facing demographic challenges. The United Nations Development Programme in Russian Federation, Moscow.

Wagle, J., Farner, L., Flekkøy, K., Bruun Wyller, T., Sandvik, L., Fure, B., . . . Engedal, K. (2011). Early post-stroke cognition in stroke rehabilitation patients predicts functional outcome at 13 months. Dementia and Geriatric Cognitive Disorders, 31, 379-387. http://dx.doi.org/10.1159/ 000328970

Wierzbicka, A. (1997). Understanding cultures through their key words: English, Russian, Polish, German, and Japanese (Vol. 8). New York, NY: Oxford University Press.

Xing, S., Lacey, E. H., Skipper-Kallal, L. M., Jiang, X., Harris-Love, M. L., Zeng, J., \& Turkeltaub, P. E. (2016). Right hemisphere grey matter structure and language outcomes in chronic left hemisphere stroke. Brain: A Journal of Neurology, 139(Pt 1), 227-241. http://dx.doi .org/10.1093/brain/awv323

Yakhno, N. N., Levin, O. S., \& Damulin, I. V. (2001). Comparison of clinical and MRI data in dyscirculatory encephalopathy. Pt. 2: Cognitive disorders. Nevrologicheskii Zhurnal, 6, 10-18.

Zwecker, M., Levenkrohn, S., Fleisig, Y., Zeilig, G., Ohry, A., \& Adunsky, A. (2002). Mini-Mental State Examination, cognitive FIM instrument, and the Loewenstein Occupational Therapy Cognitive Assessment: Relation to functional outcome of stroke patients. Archives of Physical Medicine and Rehabilitation, 83, 342-345. http://dx.doi.org/10.1053/ apmr.2002.29641

Zygouris, S., \& Tsolaki, M. (2015). New technologies and neuropsychological evaluation of older adults: Issues and challenges. In P. D. Bamidis, I. Tarnanas, L. Hadjileontiadis, \& M. Tsolaki (Eds.), Handbook of research on innovations in the diagnosis and treatment of dementia (pp. 1-17). Hershey, PA: IGI Global.

Received December 21, 2017

Revision received June 30, 2018 Accepted July 2, 2018 


\section{AUTHOR QUERIES}

\section{AUTHOR PLEASE ANSWER ALL QUERIES}

AQau-Please confirm the given-names and surnames are identified properly by the colors. = Given-Name, $\boldsymbol{a}=$ Surname The colors are for proofing purposes only. The colors will not appear online or in print.

AQ1-Author: Please be sure to provide the name of the department(s) with which you and your coauthors are affiliated at your respective institutes if you have not already done so. If you are affiliated with a governmental department, business, hospital, clinic, VA center, or other nonuniversity-based institute, please provide the city and U.S. state (or the city, province, and country) in which the institute is based. Departments should be listed in the author footnote only, not the byline. If you or your coauthors have changed affiliations since the article was written, please include a separate note indicating the new department/affiliation: [author's name] is now at [affiliation].

AQ2-Author: Your article contains supplemental material. If you have queries for references that are not cited in the text, these can be ignored if the references are found in the supplemental material.

AQ3-Author: There is no Carson et al., 2000 on the reference list. Please resolve.

AQ4-Author: There is no Easton et al., 2009 on the reference list. Please resolve.

AQ5-Author: There is no Hachinski et al., 2006 on the reference list. Please resolve.

AQ6-Author: Please spell out NINDS-CSN VCI.

AQ7-Author: I've edited the name of this agency to match what it is called most frequently. Please confirm.

AQ8 - Author: The "Instructions" subheading has been deleted per APA style (i.e., there should be no lone subheadings within a given section) and because it was not necessary. Please confirm/ approve.

AQ9-Author: There is no Mukher \& Patil, 2011 on the reference list. Please resolve.

AQ10-Author: Please provide the publisher's city/state/province location.

AQ11-Author: Please provide publisher city/state/province location.

AQ12-Author: Please provide URL. 


\section{AUTHOR QUERIES}

\section{AUTHOR PLEASE ANSWER ALL QUERIES}

AQ13-Author: This reference list entry is incomplete. Please provide missing publication information.

AQ14-Author: Please provide city/state location of publisher.

AQ15-Author: This reference list entry is incomplete. Please provide missing publication information. 